\title{
PHOTON-COUNTING MULTIKILOHERTZ MICROLASER ALTIMETERS FOR AIRBORNE AND SPACEBORNE TOPOGRAPHIC MEASUREMENTS
}

\author{
John J. Degnan \\ Geoscience Technology Office, Code 920.3 \\ NASA Goddard Space Flight Center \\ Greenbelt, MD 20771 USA
}

\begin{abstract}
We consider the optimum design of photon-counting microlaser altimeters operating from airborne and spaceborne platforms under both day and night conditions. Extremely compact Qswitched microlaser transmitters produce trains of low energy pulses at multi-kHz rates and can easily generate subnanosecond pulsewidths for precise ranging. To guide the design, we have modeled the solar noise background and developed simple algorithms, based on Post-Detection Poisson Filtering (PDPF), to optimally extract the weak altimeter signal from a high noise background during daytime operations. Practical technology issues, such as dector and/or background during daytime operations. Practical tech an airborne
receiver dead times, have also been considered in the analysis. We describe an airbor
prototype, being developed under NASA's Instrument Incubator Program, which is designed to operate at a $10 \mathrm{kHz}$ rate from aircraft cruise altitudes up to $12 \mathrm{~km}$ with laser pulse energies on the order of a few microjoules. We also analyze a compact and power efficient system designed to operate from Mars orbit at an altitude of $300 \mathrm{~km}$ and sample the Martian surface at rates up to 4.3 $\mathrm{kHz}$ using a lwatt laser transmitter and an $18 \mathrm{~cm}$ telescope. This yields a Power-Aperture Product of $0.24 \mathrm{~W}-\mathrm{m}^{2}$, corresponding to a value almost 4 times smaller than the Mars Orbiting Laser Altimeter $\left(0.88 \mathrm{~W}-\mathrm{m}^{2}\right)$, yet the sampling rate is roughly 400 times greater $(4 \mathrm{kHz}$ vs $10 \mathrm{~Hz})$.

Relative to conventional high power laser altimeters, advantages of photon-counting laser altimeters include: (1) a more efficient use of available laser photons providing up to two orders

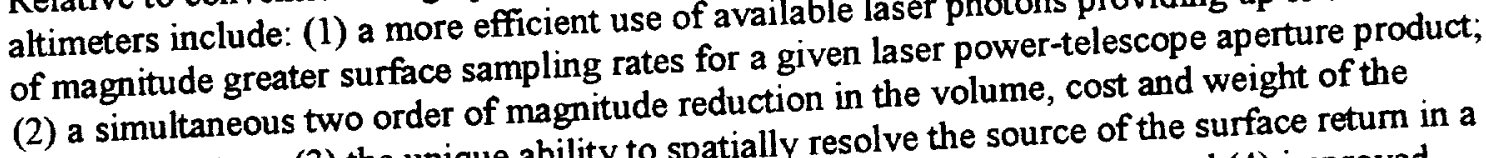
telescope system; (3) the unique ability to spatially resolve the source of the surface return in photon counting mode through the use of pixiting from both (1) and (3). Furthermore, because of vertical and transverse spatial resolution resulting from significantly lower laser pulse energies, the microaltimeter is inherently
observers on the ground and less prone to internal optical damage, which can terminate a space mission prematurely.
\end{abstract}




\section{INTRODUCTION}

Spaceborne laser altimeters typically use modest energy (50 to $100 \mathrm{~mJ}$ ) solid state lasers, large telescopes (50 to $100 \mathrm{~cm}$ diameter), and high detection thresholds to achieve unambiguous surface returns with few or no "false alarms" resulting from solar background noise [Abshire et al, 2000; Dubayah et al, 1997; Ramos-lzquierdo et al, 1994]. As a result of this conventional design philosophy, spacecraft prime power and weight constraints typically restrict operations to low repetition rates on the order of a few tens of $\mathrm{Hz}$ which, at typical planetary orbital ground velocities of a few $\mathrm{Km} / \mathrm{sec}$, limits along-track spatial sampling to one sample every few hundred meters. There is great scientific interest in obtaining higher along-track resolution and/or better cross-track coverage, but achieving this capability through a simple scaling of the laser fire rate (power) is not practical. This is especially true of altimeters destined for orbit about distant planets where instrument mass and prime power usage is severely constrained.

We will demonstrate in this paper that the conventional high Signal-to-Noise Ratio (SNR) approach to laser altimetry does not make efficient use of the available laser photons. The surface return rate of an orbiting altimeter can be increased by up to two orders of magnitude for a given laser output power by emitting the available photons in a high frequency (few $\mathrm{KHz}$ ) train of low energy $(<1 \mathrm{~mJ})$ pulses, as opposed to a low frequency train of high energy pulses, and by employing single photon detection [Degnan and McGarry, 1998]. This mode of operation reduces the risk of internal optical damage to the laser, thereby improving long-term reliability, and makes the beam inherently more eyesafe to a ground-based observer. In addition, these high return rates can be accomplished with much smaller telescope apertures on the order of 10 to 20 $\mathrm{cm}$ diameter. Indeed, the contrast of the terrain "signal" against the solar-induced noise background is actually enhanced through the use of small receive telescopes. As we will demonstrate, relatively simple onboard software algorithms, loosely based on post-detection Poisson filtering techniques, previously used in lunar laser ranging [Abbott and Shelus, 1973], can be employed to identify and extract the surface sampling data from the more abundant background noise prior to onboard data storage or transmission of the data to a ground station.

The near order of magnitude reduction in telescope diameter greatly simplifies the mechanics of scanned systems and allows the use of relatively inexpensive, modest diameter optical wedges or holographic optical elements to simultaneously scan both the transmit and receive beams for cross-track interrogation of the terrain while still maintaining narrow fields of view for background noise suppression. In addition, since the volume and weight of a telescope and its support structure nominally varies as $D^{2.3}$, where $D$ is the telescope diameter [Minott, 1998], a two order of magnitude reduction in weight and volume and a comparable reduction in fabrication cost can be realized.

Further unique performance enhancements are also possible when operating in a photon-counting mode. Commercial and developmental photon-counting detectors now exist which are capable of providing centimeter level ranging resolution as well as angularly resolving the source of a single photon event within the receiver field-of-view. These devices can be realized either as specially designed high bandwidth photomultipliers [Charboneau et al, 1992; Ebstein et al, 1996; Priedhorsky et al, 1996] or as Avalanche PhotoDiode (APD) arrays [Vasile et al, 1997]. With high angular resolution of the single photon source, the measured range becomes nearly a pointto-point measurement, i.e. from the intemal altimeter reference point to a small area of uncertainty on the surface, which is determined by the angular resolving power of the photodetector and can be very small compared to the total beam area on the ground. The ranging 
precision is now limited by the laser pulsewidth, the timing capabilities of the range receiver, and the much-reduced residual spreading of the retum waveform , or "Poisson generating function" in photon counting mode, caused by surface roughness and slope within this small zone of uncertainty. This ability to measure the near "point-to-point time-of-flight" of an individual photon removes much of the range ambiguity inherent in current high energy altimeters. In the latter, multiple photons reflected from anywhere within the illuminated spot must be recorded by power-hungry waveform digitizers and deconvoluted using complex (and often fallible) algorithms in order to decipher the results and obtain a single range measurement.

We believe that the proposed microlaser altimeter, or microaltimeter, can address many of the same Earth science issues as NASA's Earth Observing System (EOS) Geoscience Laser Altimetry System (GLAS) [Abshire et al, 2000] and Vegetation Canopy Lidar (VCL) missions [Dubayah et al, 1997]. It is also an attractive successor to the highly successful Mars Orbiting Laser Altimeter (MOLA) instrument [Ramos-Izquierdo et al, 1994], which is currently mapping the surface topography of Mars with unprecedented accuracy from the Mars Global Surveyor (MGS) satellite. Potential advantages of the microaltimeter approach include significantly greater spatial resolution in either the along-track or cross-track directions (or both) as well as greatly reduced demands on spacecraft resources such as prime power, volume and weight allocations, etc. This will be demonstrated in analysis and simulations presented later. Potential microaltimeter targets are the usual land, ice, and water surfaces as well as distributed or soft targets such as clouds, planetary boundary layers, tree canopies, and other vegetation [Ho and Priedhorsky, 1995]. The much-reduced signal levels of the microaltimeter relative to conventional altimeters are largely offset by a corresponding reduction in the detection threshold to about one photoelectron so that instrument sensitivity is largely maintained or even enhanced. As a result, geoscience applications include the development of a high resolution, high accuracy topographic database of land surfaces useful for studying hydrological runoff; the effects of clouds on radiation balance; changes in sea, lake, or reservoir levels; changes in ice sheet thickness; tree canopy heights and biomass assessment, etc. Applications to extraterrestrial science missions are both obvious and numerous and include the low power, high resolution topographic mapping of other planets, moons, asteroids, and comets within the Solar System. Commercial applications include aerial surveying of cities and towns and/or the generation of local topographic maps from aircraft cruise altitudes ( 7 to $12 \mathrm{~km}$ ). Even with their higher pulse energies, conventional airbome laser altimeters typically must fly at altitudes below $3 \mathrm{~km}$, which usually requires a special waiver from government aircraft authorities.

In Section 2 and the appendices, we develop the theory of operation for the microaltimeter Section 3 provides a brief technical description and performance simulations of a $10 \mathrm{kHz}$ airborne microaltimeter currently being developed under NASA's Instrument Incubator Program. Section 4 applies the theory to the design of a multi-kilohertz microaltimeter orbiting Mars, and Section 5 provides a brief summary of the results.

\section{THEORY OF OPERATION}

\subsection{PHYSICAL MOTIVATION FOR THE "MICROALTIMETER"}

The mean number of signal photoelectrons detected per laser fire by the receiver from a planar Lambertian diffuse reflector which fills the receiver field of view (FOV) is given by the familiar altimeter equation [Stitch, 1972]. 
$n_{s}=\left[\rho \cos \sigma T_{0}^{2}\right] \frac{\eta_{q} \eta_{r} E_{t} A_{r}}{\pi h v R^{2}}=C_{a} \frac{E_{t} A_{r}}{R^{2}}=C_{a} \frac{P_{t} A_{r}}{f_{Q S} R^{2}}$

where $C_{a}$ is the altimeter constant, $h v$ is the laser photon energy, $\eta_{q}$ is the detector quantum efficiency, $\rho$ is the surface reflectivity, $\eta_{r}$ is the transmission efficiency of the receiver optics, $\sigma$ is the surface slope, $E_{T}$ is the transmitted energy, $A_{r}$ is the collecting area of the receiver, $R$ is the range to the surface, $f_{Q S}$ is the Q-switching frequency, $P_{t}$ is the transmitter power, and $T_{0}$ is the one-way atmospheric transmission along the altimeter nadir path given by

$$
T_{0}=\exp \left(-\int_{h_{s}}^{\infty} d z \mu(z)\right)
$$

where $h_{s}$ is the height of the surface above sea level and $\mu(z)$ is the altitude-dependent atmospheric extinction coefficient. Solving for $f_{Q S}$ in (la) yields

$$
f_{Q S}=\left[\rho \cos \sigma T_{0}^{2}\right] \frac{\eta_{q} \eta_{r} P_{T} A_{R}}{n_{s} \pi h v R^{2}}
$$

If we assume that the receiver integrates over the entire surface return waveform, the frequency of range returns per second is obtained by multiplying (2) by the probability of detection for a Poisson process, i.e.

$$
f_{R}=f_{Q S} P_{D}\left(n_{s}, n_{t}\right)=f_{\max } \frac{1}{n_{s}}\left(1-e^{-n_{s}} \sum_{k=0}^{n_{t}-1} \frac{n_{s}^{k}}{k !}\right)
$$

where $n_{t}$ is the receiver detection threshold in photoelectrons, and the maximum surface return rate is defined by

$$
f_{\max }=\left[\rho \cos \sigma T_{0}^{2}\right]\left[\frac{\eta_{q} \eta_{r} P_{T} A_{R}}{\pi h v R^{2}}\right]
$$

where we have divided the latter expression into two factors, a target/atmosphere dependent term and a term which depends solely on instrumental characteristics. A normalized plot of Eq. (3) in Figure 1 demonstrates that, for a given laser power - receive aperture product, the maximum surface return rate is obtained by adopting a single photon detection threshold $\left(n_{\tau}=1\right)$ and a low mean signal strength, i.e. $n_{s}<0$. 1 . Physically, this implies using a high repetition rate, but low energy, laser transmitter, but clearly the effects of background noise must be taken into account in systems designed to operate under both day or night conditions from orbit. Our prototype airborne system, described in Section 3, proposes to use gated visible detectors, which are typically characterized by relatively low dark count rates, and the frequency-doubled $\mathrm{Nd}$ :YAG wavelength of $532 \mathrm{~nm}$. Thus, scattered solar radiation, both off the viewed surface and the the dominant source of instrument noise during daylight operations. 


\subsection{CORRELATION RANGE RECEIVER}

A correlation range receiver (CRR) can be used to distinguish weak signals from background noise counts during daylight operations [Degnan and McGarry, 1997]. The CRR measures photon time-of-flight at multi-kilohertz rates relative to the outgoing pulse, and, in spaceborne or very high repetition rate airbome applications, must be able to handle multiple pulses in flight. With each laser fire, the CRR is gated to accept only those photons which enter the receiver during a time period in which surface retums are expected. It can also record multiple photon "stop" events which occur within the range gate. Software within the CRR breaks each range gate (along the vertical range axis) into a set of equal duration range bins as in Figure 2. A set of sequential laser fires along the horizontal (time) axis forms a frame. The range bin width, $\tau_{b}$, in the CRR is chosen to accommodate all likely signal photon arrival times from a single surface occurring within a frame as defined by a frame sampling interval, $\tau_{j .}$. The 2-D area defined by the horizontal range bin and vertical frame boundaries respectively in Figure 2 is called a cell. A set of $M$ contiguous frames is a superframe. Figure 2 shows a superframe consisting of 10 frames. The terrain is shown as a solid black line passing through the various cells.

Software in the CRR tentatively identifies the signal cell by summing the counts in each cell and comparing the total to a frame threshold value, $\mathrm{K}_{\text {opt }}$, which is optimally chosen based on Poisson statistics (see Section 2.5). If the count exceeds the threshold, the cell is tentatively identified as containing signal (the gray cells in Figure 2); otherwise, it is tentatively identified as containing noise (the white cells in Figure 2). Figure 2 also illustrates a situation where a steeper than average surface slope or highly modulated terrain causes the signal counts in a frame to fall into two or more cells with the result that none of these cells achieve frame threshold as in frames 3 , 7 , and 8 . It is also statistically possible that a noise cell is occasionally mistakenly identified as signal. Under these weaker signal to noise conditions, further confirmation of signal acquisition can be obtained, if necessary, by applying an $N$ of $M$ test [Titterton et al, 1998] to the data which requires that, in $N$ of $M$ successive frames making up a superframe, a cell (1) passes the threshold test and (2) is displaced from signal cells in adjacent frames by no more than one range bin in the vertical (range) direction. We refer to this condition as a valid trajectory. A sample of a valid trajectory, in which the signal cells exceed threshold in 7 of 10 frames, is also illustrated in Figure 2.

More sophisticated tracking algorithms, which look forward and backward along the time axis, are also possible and can further improve the accommodation of steep or rapidly modulated terrain features and recover missing data. For example, looking forward in time and using our adjacent range bin criteria, the lost signal in frame 3 is expected to occur in bin 4,5, or 6 (cells with upwardly sloped lines) since bin 5 was identified as the signal in Frame 2. However, looking backward in time from frame 4 suggests that bins 6,7 , and 8 are the likely candidates in Frame 3 (cells with downwardly sloped lines). The only cell common to both the forward and backward groupings in Frame 3 is bin \#6 (cross-hatched), which in reality contains most of the signal.

As a second example, we consider Frames 7 and 8 where the signal cell goes undetected in two consecutive frames. Looking forward in time from Frame 6 suggests the signal may be in bins 6 consecutive frames. Looking forward in time from Frame 6 suggests the 5 through 9 in Frame 8 . Looking backwards in time from Frame 9 ,
through 8 in frame 7 and bins 5 throw 7 in Frame 7 . The overlapping
however, suggests bins 4 through 6 in Frame 8 and 3 through 7 in the (cross-hatched) bins are 6 and 7 in Frame 7 and bins 5 and 6 in Frame 8, which indeed contain the missing signal counts. While very abrupt changes in terrain (such as a steep cliff) can cause the signal cell to jump many range bins in a single frame and violate our \pm one bin criteria, we will 
demonstrate in later simulations that the algorithm quickly identifies the surface at the new elevation.

Surface slope and/or roughness within the interrogated surface area, $A_{s}$, will produce a return waveform (or, in the case of photon counting, a Poisson generating function) that is significantly broadened relative to the initial laser pulsewidth, which is typically on the order of a nanosecond. Distributed targets, such as clouds or ground vegetation, also produce broadened returns and, with a properly designed receiver, are detectable using photon counting instruments [Ho and Priedhorsky, 1995]. Multiple returns from different entities - e.g. cloudtops, tree canopies, and/or ground returns - can also occur on a single laser pulse, potentially causing several CRR cells, widely separated in range, to record valid retums.

\subsection{SOLAR NOISE BACKGROUND}

From equations (A7) and (A9) in Appendix A, the mean number of background noise counts generated within a given range bin by scattered solar radiation is given by

$n_{b}=\beta A_{r} \tau_{b}=\left[N_{\lambda}^{0}(\Delta \lambda) \Omega_{r} \tau_{b}\right]\left[\frac{\eta_{Q} \eta_{r} A_{r}}{\pi h \nu}\right]\left[\rho T_{0}^{1+\sec \theta_{s}} \cos \psi+\frac{1-T_{0}^{1+\sec \theta_{s}}}{4\left(1+\sec \theta_{s}\right)}\right]$

where the first and second terms respectively correspond to the noise counts per range bin due to scattered solar radiation from the surface under study and the intervening atmosphere. In Eq. (5), which is a generalization of Eq. (18) in [Stitch, 1972], $\beta$ is the combined noise count rate per unit area of receive aperture, $N_{\lambda}^{0}$ is the wavelength-dependent solar spectral illuminance as measured just outside the planetary atmosphere $\left(N_{\lambda}^{0}=0.2\right.$ watts $/ \mathrm{m}^{2}-\mathrm{A}^{0}$ at $532 \mathrm{~nm}$ for Earth), $\Delta \lambda$ is the spectral filter bandpass, $\Omega_{r}$ is the the receiver field of view (FOV) in steradians, $\tau_{b}$ is the duration of the range bin, $\psi$ is the subtended angle between the Sun and the surface normal, and $\theta_{s}$ is the solar zenith angle at the time of the measurement. It is also shown in Appendix A that

$\cos \psi=\cos \sigma \cos \theta_{s}+\sin \sigma \sin \theta_{s} \cos \varphi$

where $\varphi$ is the angle between the plane containing the altimeter line-of-sight and the Sun ( $x-z$ plane) and the plane containing the altimeter line-of-sight and the surface normal as in Figure 3 Since $1 \geq \cos \varphi \geq-1$, we have the condition

$$
\cos \left(\sigma-\theta_{s}\right) \geq \cos \psi \geq \cos \left(\sigma+\theta_{s}\right)
$$

where $\cos \psi$ has the maximum value of 1 when $\varphi=0$ and $\sigma=\theta_{s}$ and a minimum value of 0 when the surface is in shadow $(\cos \psi \leq 0)$.

\subsection{INSTRUMENT CONTRAST AND SCALE ENERGY}

Using (1) and (5), we can define an instrument contrast

$$
C=\frac{N_{b}+N_{s}}{N_{b}}=1+\frac{f_{Q S} \tau_{f} n_{s}}{f_{Q S} \tau_{f} n_{b}}=1+\frac{n_{s}}{n_{b}}=1+\frac{E_{T}}{E_{s c}}
$$


where we have assumed single photoelecton detection, $N_{f}=f_{q s} \tau_{f}$ is the number of laser fires per frame, and $n_{s}$ and $n_{b}$ are the mean signal and noise counts per range bin per laser fire respectively. The contrast is equal to the ratio of total mean counts in the signal cell to the mean background counts collected within any other noise cell in the CRR. We have also defined a scale energy given by

$$
E_{s c}=\left[N_{\lambda}^{0}(\Delta \lambda) \Omega_{r} R^{2} \tau_{b} \sec \sigma\right]\left[T_{0}^{\sec \theta_{z}-1} \cos \psi+\frac{1}{4 \rho T_{0}^{2}} \frac{\left(1-T_{0}^{1+\sec \theta_{z}}\right)}{1+\sec \theta_{z}}\right]
$$

as that transmitted single pulse energy which produces the same mean signal count in a properly sized cell (i.e. one containing all of the expected mean surface returns within a frame) as the solar noise background produces in any other arbitrary cell and which in turn produces a cell contrast of 2 . The scale energy is independent of other details related to the instrument, atmosphere, or surface properties since these will have an equal scaling effect on both signal and background noise and hence will not affect the contrast. We note from (7b) that the scale energy varies quadratically with range and linearly with both the receiver solid angle FOV and the range bin duration

In the limit as $T_{0} \rightarrow 1$ (relevant to an altimeter orbiting an airless moon or asteroid), (7b) reduces to the simple expression

$E_{s c}=N_{\lambda}^{0} \Delta \lambda \Omega_{r} R^{2} \tau_{b} \sec \sigma \cos \psi \leq N_{\lambda}^{0} \Delta \lambda \Omega_{r} R^{2} \tau_{b} \sec \sigma$

Assuming a ground spot diameter of $\sim 25$ meters (corresponding to a 10 arcsecond laser beam at an orbital altitude of $500 \mathrm{Km}$ ), a modest filter bandpass of $\sim 3 \mathrm{~A}^{\circ}$, a range bin of $\sim 200 \mathrm{nsec}$, and no surface slope $(\sigma=0)$, the scale energy is less than $120 \mu \mathrm{J}$, or almost 3 orders of magnitude smaller than the $100 \mathrm{~mJ}$ GLAS transmitter pulse energy. For a relatively steep surface slope of $70^{\circ}$, the scale energy is increased by roughly a factor of 3 to $360 \mu \mathrm{J}$. We will demonstrate shortly that a transmitted energy 2 to 3 times the scale energy is usually adequate to achieve high reliability discrimination of the surface returns from the worst case solar background. Thus, Earth mapping missions using less than $1 \mathrm{~mJ}$ of transmitted energy are feasible in photon counting mode.

In order to compute the maximum scale energy in the presence of a scattering atmosphere, we will find it useful to introduce the variables

$$
\omega=\frac{1}{T_{0}^{1+\sec \theta_{z}}} \quad \frac{1}{T_{0}^{2}} \leq \omega<\infty
$$

and

$$
\alpha=\frac{1}{4 \rho \cos \psi} \ln \left(\frac{1}{T_{0}}\right)
$$

which, when substituted into (7b), yield 
$E_{s c}=\frac{N_{\lambda}^{0} \Delta \lambda \Omega_{r} R^{2} \tau_{b} \sec \sigma}{T_{0}^{2}} \cos \psi \frac{1+\alpha \frac{\omega-1}{\ln \omega}}{\omega}$

Setting the derivative of $(10)$ with respect to $\omega$ equal to zero

$\frac{d E_{s c}}{d \omega}=\frac{N_{\lambda}^{0} \Delta \lambda \Omega_{r} R^{2} \tau_{b} \sec \sigma}{T_{0}^{2}} \cos \psi\left\{\frac{-\ln ^{2} \omega+\alpha(\ln \omega-\omega+1)}{\omega^{2} \ln ^{2} \omega}\right\}=0$

suggests a single extremum at $\omega=1$. However, since $E_{s c}$ and its derivative are both indeterminate, we must make repeated use of L'Hospital's rule to obtain

$\lim _{\omega \rightarrow 1} E_{s c}=\frac{N_{\lambda}^{0} \Delta \lambda \Omega_{r} R^{2} \tau_{b} \sec \sigma}{T_{0}^{2}}\left[\cos \psi+\frac{1}{4 \rho} \ln \left(\frac{1}{T_{0}}\right)\right]$

and

$\lim _{\omega \rightarrow 1} \frac{d E_{s c}}{d \omega}=-\frac{N_{\lambda}^{0} \Delta \lambda \Omega_{r} R^{2} \tau_{b} \sec \sigma}{T_{0}^{2}}\left[\cos \psi+\frac{1}{8 \rho} \ln \left(\frac{1}{T_{0}}\right)\right]$

Since the derivative (12b) is negative, the extremum at $\omega=1$ is a maximum. Thus, substituting $\omega$ $=1 / T_{0}{ }^{2}$, the smallest possible physical value of $\omega$ in $(9 a)$, into (10) yields an upper limit for the scale energy, i.e.

$E_{s c} \leq N_{\lambda}^{0} \Delta \lambda \Omega_{r} R^{2} \tau_{b} \sec \sigma\left[1+\frac{1}{8 \rho}\left(\frac{1}{T_{0}^{2}}-1\right)\right]$

which correctly reduces to the surface-only expression (8b) as $T_{0} \rightarrow 1$. Thus, the effect of the worst case atmospheric scatter is to increase the maximum scale energy by more than a factor of 2 for low reflectivity surfaces that satisfy the condition

$\rho \leq \frac{1}{8}\left(\frac{1}{T_{0}^{2}}-1\right)$

which, for typical atmospheric transmissions at $532 \mathrm{~nm}\left(\mathrm{~T}_{0}>0.7\right)$, primarily affects surfaces with reflectivities less than about 0.13 .

2.5 DISCRIMINATING SIGNAL FROM NOISE WITH POST-DETECTION POISSON ANALYSIS

To achieve a maximum surface return rate during daylight operations of the altimeter, the transmitted energy must be sufficiently greater than the scale energy to permit successful discrimination of the surface against solar background noise within a reasonable frame interval, i.e. there must be sufficient contrast as defined by (7). On the other hand, the contrast should not be so great as to unnecessarily limit the pulse repetition rate (and hence surface sampling rate) for 
a given laser output power. In any case, we are interested in designing a CRR which can effectively identify signal cells under conditions of relatively low or modest contrast $(C \geq 3)$.

Since the system parameters must be chosen such that the mean noise count per range bin per laser fire is significantly less than the mean signal count per laser fire (which is also on the order of unity or less for efficiency reasons as previously described in Section 2.1), usually no more than one photon count (signal or noise) is accumulated in any given cell per laser fire. On the other hand, the total number of noise counts observed within the range gate per laser fire may be significantly greater than one since, although the probability of detecting a noise count in a given range bin is small, there can be a large number (hundreds to thousands) of range bins if the range gate (range uncertainty) is large. Furthermore, the requirement that the noise count per bin be much less than the mean signal count $(<1)$ places a constraint on the maximum telescope aperture that can be employed in daylight operations to reach the necessary power-aperture product for a given surface sampling rate, although this limit can be relaxed somewhat by improved spatial and spectral filtering.

From Poisson statistics, the probability that we will correctly identify and acquire the signal cell in a given frame is

$$
P_{a \alpha q}=P\left(N_{t} \geq K\right)=1-e^{-N_{t}} \sum_{k=0}^{K-1} \frac{N_{t}^{k}}{k !}=1-e^{-C N_{b}} \sum_{k=0}^{K-1} \frac{\left(C N_{b}\right)^{k}}{k !}
$$

where $N_{t}=N_{s}+N_{b}=C N_{b}$ is the total mean photoelectron count, equal to the sum of the mean signal and background counts in the cell, and $\mathrm{K}$ is the frame count threshold. Similarly, the probability of falsely identifying any given noise cell as "signal" is equal to

$$
P_{\text {false }}=P\left(N_{b} \geq K\right)=1-e^{-N_{b}} \sum_{k=0}^{K-1} \frac{N_{b}^{k}}{k !}
$$

Now, the mean number of noise cells per frame falsely registering a signal is given by

$$
N_{\text {false }}=N_{\text {bin }} P_{\text {false }}
$$

where $N_{b i n}=\tau_{g} / \tau_{b}$ is the number of range bins within the range gate. Thus, for the $M$ frames making up a superframe, we can define a differential cell count

$$
N(K) \equiv M\left[P_{a c q}-N_{b i n} P_{\text {false }}\right]=M\left\{1-e^{-C N_{b}} \sum_{k=0}^{K-1} \frac{\left(C N_{b}\right)^{k}}{k !}-N_{b i n}\left[1-e^{-N_{b}} \sum_{k=0}^{K-1} \frac{\left(N_{b}\right)^{k}}{k !}\right]\right\}
$$

which subtracts the expected mean number of falsely acquired noise cells from the expected mean number of true signal cell acquisitions occurring within a superframe.

Choosing a frame threshold $\mathrm{K}$ in (17) that is too small will result in too many noise cells being falsely identified as signal and will drive the differential cell count to small $(<<\mathrm{M})$, or even negative, values. On the other hand, choosing a threshold that is too high will reject many valid signal cells, along with the noise cells, and drive the differential cell count toward zero. We therefore pose the question - what integer frame count threshold, $K$, maximizes the differential 
cell count? To obtain the answer, we perform a quasi-differentiation with respect to the frame threshold, $\mathrm{K}$, on (17), i.e.

$$
\frac{\Delta N}{\Delta K}=N(K+1)-N(K)=\frac{N_{b}^{K} e^{-N_{b}}}{K !}\left[N_{b i n}-C^{K} e^{-(C-1) N_{b}}\right]
$$

Setting the latter expression equal to zero and solving for $\mathrm{K}$ gives a simple formula for the optimum frame threshold, $K_{\text {opt }}$

$K_{\text {opt }}=\frac{(C-1) N_{b}+\ln \left(N_{b i n}\right)}{\ln C}=\frac{N_{s}+\ln \left(N_{b i n}\right)}{\ln C}$

The threshold $K_{\text {opt }}$ maximizes the differential cell count since it can be similarly demonstrated, using (17) through (19), that the second "quasi-derivative" has a value less than zero for $C>1$, i.e.

$$
\frac{\Delta^{2} N}{\Delta K^{2}}=N\left(K_{o p t}+1\right)-2 N\left(K_{o p t}\right)+N\left(K_{o p t}-1\right)=-\frac{N_{b}^{K_{o p t}-1} C^{K_{o p t}} e^{-C N_{b}}}{\left(K_{o p t}-1\right) !}\left(1-\frac{1}{C}\right)<0
$$

One can now use (19) in (17) to eliminate $N_{b i n}$ in the expression for the optimized differential cell count and obtain

$N_{\text {opt }}=M\left\{1-C^{K_{\text {opt }}} e^{-(C-1) N_{b}}-e^{-C N_{b}} \sum_{k=0}^{K_{\text {op }}-1} \frac{\left(C^{k}-C^{K_{\text {opx }}}\right) N_{b}^{k}}{k !}\right\}$

Using (21), we can further show that, in the extreme contrast limits,

$\lim _{C \rightarrow 1} N_{o p t}=0$

and

$\lim _{C \rightarrow \infty} N_{o p t}=M$

Thus, since $N_{\text {opt }}$ is always positive for the optimized threshold, the mean number of correctly identified signal cells always exceeds the number of falsely acquired noise cells and hence the latter can never exceed a mean of one per frame or $M$ per superframe since that is the maximum number of allowed signal cells. One can substitute the optimum threshold (19) into (15) and (16) to compute the probabilities of correctly identifying the signal cell, the probability of falsely identifying a noise cell as signal, or the mean number of noise cells per frame which exceed the optimized threshold. As we will demonstrate later in the numerical analyses of airbome and spaceborne altimeters, one can usually drive the differential cell count arbitrarily close to the ideal value $M$ (i.e. there are $M$ correctly identified signal cells in a superframe and zero false acquisitions) by choosing a sufficiently long frame interval. 
If we correctly select the signal cell and reject the other cells, the fraction of noise counts retained by the instrument is $1 / N_{b i n}$. Within the signal cell, $1 / C$ of the counts are expected to be noise counts on average. In Figure 4, we plot the differential cell count (normalized to $M$ ), the probability of detection, and the mean number of false alarm cells per frame as a function of the mean number of signal counts in the frame $\left(N_{s}\right)$ for $N_{b i n}=100$ (i.e. noise retention rate $=1 \%$ ) and four values of the contrast, i.e. $\mathrm{C}=3,5,10$ and 20 . As expected, at higher contrast, fewer signal counts per frame are required to positively identify the signal cell and reduce the number of false alarms to an insignificant level. We further note from the figure that, for $N_{s}>40$ and all of the contrast values considered, the normalized differential cell count and probability of correctly detecting the signal cell are virtually indistinguishable from unity and the mean number of false alarms is less than one per thousand frames. Assuming different values for $N_{b i n}$ in the range from 20 to 500 only changes the plots in the region of small $N_{s}$ and does not significantly affect the results for large $N_{s}$. In summary, collecting about 40 signal counts per frame provides excellent discrimination over a wide range of signal cell contrasts $(C \geq 3)$.

\subsection{CHOOSING THE RANGE BIN AND FRAME INTERVAL}

The choice of range bin and frame interval are intimately interconnected. The frame duration cannot be made arbitrarily large (e.g., over a sloped surface) without eventually forcing a larger range bin $\tau_{b}$ in order to retain all of the surface returns within a single cell. As can be seen from (10), a larger range bin also implies proportionally larger scale energies, which reduces the cell contrast for a fixed mean signal strength. In a worst case situation, where we are ranging off a constant steep slope $\sigma$ for the entire duration of a frame, all of the signal counts observed during the frame will always be contained within a single cell if we choose a range bin duration which is twice as large as the total signal spread within a frame

$$
\tau_{b}=2\left[\tau_{0}+\frac{2}{c}\left(D_{g}+v_{g}^{e f f} \tau_{f}\right) \tan \sigma\right]=2 \tau_{s}+\gamma \tau_{f}
$$

where $\mathrm{c}$ is the speed of light, $D_{g}$ is the beam diameter on the ground, $v_{g}^{\text {eff }}$ is the effective ground velocity of the beam, $\tau_{f}$ is the frame duration, and $\tau_{0}$ is the minimum temporal width of the single pulse return waveform from a normal planar surface and is determined by factors such as the laser pulsewidth, beam divergence, and detector impulse response. The single shot return waveform is broadened relative to this minimum value by the local slope according to the equation

$$
\tau_{s}=\tau_{0}+\frac{2 D_{g} \tan \sigma}{c}
$$

The parameter

$$
\gamma=\frac{4 v_{g}^{e f f} \tan \sigma}{c}=\frac{4\left(v_{g}+2 \pi R f_{s c a n} \tan \xi\right) \tan \sigma}{c}
$$

is the rate at which the range bin grows with increasing frame length due to beam velocity and surface slope effects. For an unscanned system, the effective ground velocity of the beam is equal to the aircraft or spacecraft ground velocity, $v_{g}$. Since a typical ground velocity for planetary orbits is a few $\mathrm{km} / \mathrm{sec}$, the ratio $v_{g}^{e f f / c}$ in Eq. (23) suggests that the range bin duration can be between 4 to 5 orders of magnitude smaller than the frame interval for all but nearly vertical 
slopes $\left(\sigma \approx 90^{\circ}\right.$ ). For typical aircraft cruise velocities on the order of 0.1 to $0.2 \mathrm{~km} / \mathrm{sec}$, the range bin duration can be 5 to 6 orders of magnitude smaller than the frame duration.

If we include a scanning mechanism on the altimeter to obtain cross-track measurements of the underlying terrain, the effective ground velocity of the beam can be significantly larger than the ground velocity of the host vehicle, leading to an even larger range bin and a further reduction in signal cell contrast. A relatively simple, although not necessarily optimum, scanning device is a slightly wedged optical plate spun at a frequency $f_{\text {scan }}$ in front of the transmit and receive optics. Because of the small optics (typically 10 to $20 \mathrm{~cm}$ ) required for the microaltimeter, this is a viable scanning approach and produces a circular ground pattem superimposed on the linear motion of the aircraft or spacecraft. Since the frame interval is usually short compared to the circular scan period, the maximum effective ground velocity occurs when the linear ground velocity of the aircraft or spacecraft and the beam velocity due to the scan add vectorially as in (23c), where $R$ is again the range to the surface and $\xi$ is the angular deviation of the circularly-scanned beam from nadir (half angle of the cone). Two counter-rotating optical wedges in series can be used to create a variety of scan patterns, including a linear raster scan in the cross-track direction (same rotation frequency and wedge angle but starting $180^{\circ}$ out of phase in the along track direction).

\subsection{WAVELENGTH FIGURES OF MERIT}

Relative to $532 \mathrm{~nm}$, the $1064 \mathrm{~nm}$ fundamental Nd:YAG wavelength is known to have many "natural" advantages for altimetry, such as generally higher reflectance from various types of soil and vegetation, improved atmospheric transmission, and reduced solar background noise. Furthermore, one pays an additional penalty $(-50 \%)$ in converting infrared photons to the visible via passive nonlinear crystals. However, it will now be shown, via two instrument figures of merit (FOMs), that the much higher detector quantum efficiencies and narrower, high throughput spectral filters available at $532 \mathrm{~nm}$ often makes the latter wavelength a better choice for the microaltimeter.

\begin{tabular}{|l|l|l|l|l|c|l|l|l|c|}
\hline$\lambda(\mathrm{nm})$ & $\eta_{q}$ & $T_{0}^{2}$ & $\eta_{s h}$ & $\begin{array}{l}\rho \\
\text { soil }\end{array}$ & $\begin{array}{l}\rho \\
\text { dry veg }\end{array}$ & $\begin{array}{l}\rho \\
\text { green } \\
\text { veg }\end{array}$ & $\begin{array}{l}\text { FOM } \\
\text { soil }\end{array}$ & $\begin{array}{l}\text { FOM } \\
\text { dry veg }\end{array}$ & $\begin{array}{l}\text { FOM } \\
\text { green } \\
\text { veg }\end{array}$ \\
\hline $\begin{array}{l}1064 \\
\text { (APD) }\end{array}$ & .02 & .88 & 1 & .25 & .36 & .67 & .005 & .006 & .012 \\
\hline $\begin{array}{l}532 \\
\text { (PMT) }\end{array}$ & .15 & .64 & .5 & .15 & .15 & .1 & .004 & .004 & .003 \\
\hline $\begin{array}{l}532 \\
\text { (APD) }\end{array}$ & .40 & .64 & .5 & .15 & .15 & .1 & .010 & .010 & .006 \\
\hline
\end{tabular}

Table 1: Return Rate FOM as a function of wavelength for various surface types and detectors and a clear atmosphere. Note that, except for green vegetation, the natural advantages of the $1064 \mathrm{~nm}$ wavelength with regard to surface reflectance and atmospheric transmission are largely overcome by the significantly higher quantum efficiency of detectors at $532 \mathrm{~nm}$.

The Return Rate FOM is the wavelength-dependent portion of Eq. (4), i.e.

$F O M_{R R}=\lambda \eta_{q} \rho T_{0}^{2} \eta_{s h}$ 
where $\lambda$ is the wavelength, $\eta_{q}$ is the detector quantum efficiency, $\rho$ is the surface reflectance, $T_{0}$ is the one-way atmospheric transmission at nadir, and $\eta_{\mathrm{sh}}$ is the second harmonic generation efficiency (nominally 50\%). Table 1 shows the value of the Return Rate FOM at both wavelengths for a variety of scatterers (soil, dry vegetation, and green vegetation) and different detector types, such as a Photomultiplier (PMT) and the more efficient Geiger-mode Avalanche Photo-Diode (APD).

Similarly, the Contrast FOM is given by the wavelength-dependent portion of (7), i.e.

$$
F O M_{C}=\frac{\eta_{s h}}{N_{\lambda}^{0}(\Delta \lambda) \lambda^{2}\left[1+\frac{1}{8 \rho}\left(\frac{1}{T_{0}^{2}}-1\right)\right]}=\frac{\eta_{s h} \zeta}{N_{\lambda}^{0}(\Delta \lambda) \lambda^{2}}
$$

where $\Delta \lambda$ is the spectral filter FWHM bandwidth, the factor of $\lambda^{2}$ accounts for the reduced beam divergence at smaller wavelengths for a fixed transmit aperture, and the parameter $\zeta$ includes the combined effects of surface reflectance and atmospheric transmission.

\begin{tabular}{|l|c|c|c|c|c|c|c|c|c|}
\hline$\lambda(\mathrm{nm})$ & $N_{\lambda}{ }^{\circ}$ & $\begin{array}{l}\Delta \lambda \\
\text { ang }\end{array}$ & $\eta_{\text {sh }}$ & $\begin{array}{l}\zeta \\
\text { soil }\end{array}$ & $\begin{array}{l}\zeta \\
\text { dry veg }\end{array}$ & $\begin{array}{l}\zeta \\
\text { green } \\
\text { veg }\end{array}$ & $\begin{array}{l}\text { FOM } \\
\text { soil }\end{array}$ & $\begin{array}{l}\text { FOM } \\
\text { dry veg }\end{array}$ & $\begin{array}{l}\text { FOM } \\
\text { green } \\
\text { veg }\end{array}$ \\
\hline $\begin{array}{l}1064 \\
(\mathrm{APD})\end{array}$ & .06 & 8.0 & 1 & .94 & .95 & .98 & 2.0 & 2.0 & 2.1 \\
\hline $\begin{array}{l}532 \\
(\mathrm{PMT})\end{array}$ & .20 & 1.5 & .5 & .68 & .68 & .59 & 4.6 & 4.6 & 4.0 \\
\hline $\begin{array}{l}532 \\
(\mathrm{APD})\end{array}$ & .20 & 1.5 & .5 & .68 & .68 & .59 & 4.6 & 4.6 & 4.0 \\
\hline
\end{tabular}

Table 2: Contrast FOM as a function of wavelength for various surface types and detectors. Note that the natural advantages of the $1064 \mathrm{~nm}$ wavelength with regard to surface reflectance and solar background are more than overcome by the improved beam divergence and narrower spectral filters available at $532 \mathrm{~nm}$.

Table 2 illustrates that the Contrast FOM is roughly a factor of two higher at $532 \mathrm{~nm}$, due to the narrower spectral filters that can be fabricated and the narrower beam divergences for a given transmit aperture. Except for green vegetation, the Retum Rate FOM is comparable for both wavelengths if one uses a nominal PMT but is about twice as large at $532 \mathrm{~nm}$ if one takes advantage of higher APD quantum efficiencies in the visible. Therefore, for altimeters orbiting planets other than Earth, $532 \mathrm{~nm}$ would appear to be the wavelength of choice.

\subsection{EFFECTS OF DETECTOR AND/OR RECEIVER “DEAD-TIME"}

Following detection, real detectors/timing receivers usually experience a dead time, typically ranging from a few to hundreds of nanoseconds, during which no further count will be detected. Since signal photons from a high reflectance surface are bunched together tightly in time (often within a few nsec), any dead time which is long compared to the return waveform would be expected to limit the number of detected signal photons to one per laser fire (see Appendix B). The corresponding cell contrast would therefore be much reduced relative to that predicted by Eq (7a) for mean signal strengths substantially greater than unity. 
Different photon-counting detectors have vastly different dead time characteristics. Avalanche Photo-Diodes (APD's) operated in the so-called Geiger-mode (also known as a Single

Photoelectron Avalanche Diode or SPAD) trigger off a single photon and, following avalanche, must be restored via active or passive quenching techniques [Cova et al, 1996]. The saturated output of a SPAD is suitably shaped for direct input into an event timer without an intervening timing discriminator, and hence the effective dead time is the longer of the timing receiver dead time or the detector quenching time which is typically 10 to 40 nsec depending on APD thickness [Cova et al, 1996]. Since a photon entering a MicroChannel Plate Photo-Multiplier Tube (MCP/PMT) only depletes the charge in a few microchannels in the proximity of the cathode entry point, other photons (signal or noise) can be detected and amplified by the vast number of remaining unsaturated channels. Similarly, conventional dynode chain PMT's usually require the input of many photons to saturate the output. Hence, most photomultipliers have virtually no internal dead-time, and the effective dead-time will generally be governed by the burst-mode characteristics of the timing receiver.

It is demonstrated in Appendix B, that if the detector/receiver deadtime, $\tau_{d}$, is longer than the return waveform from the surface but shorter than the range bin duration $\left(\tau_{b}>\tau_{d},>\tau_{s}\right)$, the expression for the minimum signal cell contrast becomes

$C_{d}=1+\left(1-e^{-n_{s}}\right)\left[\frac{1}{n_{b}}-\frac{\tau_{d}}{\tau_{b}}\right]$

In (26), both the signal and noise counts per range bin, $n_{s}$ and $n_{b}$, depend linearly on the receive aperture, $A_{R}$. In the small aperture (signal) limit, the contrast approaches a maximum value

$$
\lim _{A_{R} \rightarrow 0} C_{d}=1+\frac{n_{s}}{n_{b}}
$$

equal to the zero dead time result in (7a). However, in the large aperture limit, we obtain a contrast less than unity, i.e.

$$
\lim _{A_{R} \rightarrow \infty} C_{d}=1-\frac{\tau_{d}}{\tau_{b}}
$$

suggesting that, if we choose too large a telescope, the signal will be indistinguishable from the noise regardless of the transmitted energy.

In designing a practical instrument (see Section 3), we will often choose $n_{s} \geq 1$ in order to get a high probability of detection per laser fire in single photon mode, making the exponential in (26) small compared to unity. Thus, in the high signal limit, (26) reduces to

$$
\lim _{n_{s} \rightarrow \infty} C_{d}=1-\frac{\tau_{d}}{\tau_{b}}+\frac{1}{n_{b}}
$$


As anticipated, comparing (29) with (27) demonstrates that the receiver effectively detects at most one photon in the signal swarm as a consequence of having a detector/receiver dead time longer than the return waveform. Any additional signal photons impinging on the detector are therefore wasted. Eq.(29) further indicates the importance of maintaining a background noise count per range bin, $n_{b}$, significantly less than unity.

Solving for $n_{b}$ in (27) leads to an expression for the receiver area that maintains the desired contrast, i.e.

$$
A_{R}=\frac{1}{\beta\left[\tau_{b} \frac{\left(C_{d}-1\right)}{1-e^{-n_{s}}}+\tau_{d}\right]}
$$

where $\beta$ is the maximum background noise rate per unit area per unit time defined in (5).

Thus, reducing the receiver spectral bandwidth and spatial FOV to the maximum extent possible is an imperative first step in designing an efficient photon-counting altimeter. This avoids unnecessarily constraining the receive aperture and placing more of a burden on the laser transmitter to achieve the required power-aperture product for a given mission. As we will demonstrate in Section 4, the fundamental instrument parameters (transmitter energy, receive aperture, detector $Q E$, etc.) must be chosen based on achieving the required sampling rate from a surface having a nominal worst case reflectance and slope. Over higher reflectance terrain or more modest slopes, the sampling rate will approach a maximum determined by the laser fire rate, $f_{Q S}$. We will demonstrate in later analyses that, over highly reflective terrain (e.g., snow or ice), the increased solar flux from the surface can cause the noise count per range bin to rise to unacceptable levels, leading to a severe reduction in signal cell contrast (due to receiver dead time effects) in spite of the correspondingly higher signal levels. However, this can be accommodated by the inclusion of an actively controlled attenuator in the receiver to maintain system contrast and mimic the returns from a lower reflectivity surface.

\subsection{DETECTOR ARRAYS AND SEGMENTED ANODE PHOTOMULTIPLIERS}

Let us now assume that the terrain within the receiver FOV is imaged onto $N_{p}$ pixels of a photoncounting "array" detector and that each of the $N_{p}$ pixels is fed into a separate timing channel as in the airborne system to be described in Section 3 of this paper. Possible detector configurations include an array of Geiger-mode APD's, perhaps with a microlens array in front to reduce the amount of dead space in the detector plane, or alternatively by segmenting the anode of a conventional dynode-chain or microchannel plate photomultiplier [Degnan and McGarry, 1997]. One advantage is that each pixel is now monitoring the returns/solar noise from a much smaller area on the ground, i.e. $A_{p}=A_{F O v} / N_{p}$. Furthermore, multiple signal counts can now be recorded since the detector and range receiver dead times in different timing channels are decoupled. Even if the detector elements are fed to a common event timer, a fixed time delay between channels can be introduced to overcome timer dead times and later removed from the range measurement in software while still preserving the identity of the active pixel [Degnan and McGarry, 1997].

Since the receiver response is generally flat over the FOV, the noise counts are expected to be fairly uniformly distributed over the $N_{p}$ pixels or anode segments. The transmitted laser beam, on the other hand, typically illuminates a somewhat smaller region within the receiver FOV and is 
most heavily concentrated near the center of the beam. The signal counts therefore will fall nominally on $N_{l}$ pixels where $N_{l} \leq N_{p}$. Thus, in the small aperture limit, the cell contrast in a given signal pixel channel (or the collective sum of all the active signal channels) is improved relative to the single element detector by roughly a factor of $N_{p} N_{l}$ as can be seen from (27). Of course, if all of the independent channels within the receiver FOV are "ORed" together in the receiver prior to computing the cell counts, the cell contrast is unchanged, relative to the single element detector, in the small signal/small aperture limit.

In the high signal limit $\left(n_{s}>>N_{l}\right)$, however, we see from (29) that, relative to the single element detector, the cell contrast for an individual signal channel is increased by roughly a factor of $N_{p}$ due to the fact that the solar noise counts are now shared equally by the $N_{p}$ detector elements within the receiver FOV but each of the $N_{l}$ pixels viewing the ground laser spot is still capable of detecting only one signal photon due to receiver dead time effects. Furthermore, up to $N_{l}$ individual photon TOF's can be recorded in a single laser fire interval, resulting in a single shot quasi-imaging capability provided, of course, that there are sufficient signal photons impinging on the detector. Thus, the instrument experiences an overall increase in contrast given by

$$
\lim _{n_{s} \rightarrow \infty} C_{d}=1-\frac{\tau_{d}}{\tau_{b}}+\frac{N_{l}}{n_{b}}
$$

This suggests that an instrument providing approximately one photon per active pixel from the lowest reflectance surface of interest will, from a power efficiency standpoint, be near-optimum for rapid topographic mapping. We will demonstrate this for a Mars topographic mapper in Section 4.

Solving for $n_{b}$ in (31) demonstrates that use of a pixellated detector in the high signal limit allows the receive aperture to be increased by roughly a factor of $N_{l}$ relative to the single element case in (30) while maintaining a constant contrast, i.e.

$$
A_{R}=\frac{N_{l}}{\beta\left[\tau_{b} \frac{\left(C_{d}-1\right)}{1-e^{-n_{s}}}+\tau_{d}\right]}
$$

A further important advantage of the array detector is that the broadening of the return waveform (Poisson generating function) per pixel by beam divergence or surface slope effects is reduced by roughly a factor of $1 / \sqrt{ } N_{l}$. This, combined with the ability to more accurately define the source of the returning photon within the ground spot, results in a more accurate "quasi-point-to-point" range measurement.

While they offer high angular resolving power for locating the photon source, imaging photomultipliers of the type discussed in the Introduction cannot be viewed as pixellated or segmented detectors. They have a single timing output, and the angular source of the photon in the far field is determined by additional circuitry. Thus, these devices must be treated as single element detectors, i.e. $N_{l}=1$. 


\section{THE NASA AIRBORNE MULTIKILOHERTZ MICROLASER ALTIMETER}

\subsection{PROGRAM BACKGROUND AND GOALS}

Under the auspices of NASA's Instrument Incubator Program (IIP), the Goddard Space Flight Center is developing an airborne version of the microaltimeter as a stepping stone to future space missions. Instrument development was initiated in December 1998 and the first calibration flights are scheduled for January 2001. The goals of the IIP program are to:

- Develop the necessary technologies and the operational and analytical software.

- Confirm the validity of the mathematical modeling and surface data extraction algorithms.

- Collect high spatial resolution topographic data over a variety of surfaces (land, ice, water, biomass, clouds) from a high altitude aircraft under both night and day conditions and evaluate its scientific usefulness relative to data from conventional altimeters.

- Demonstrate the technical and economic advantages of the microaltimeter concept for future Earth and interplanetary missions.

In order to improve the compatibility and portability of the instrument between different host aircraft, the microaltimeter has been designed to attach to a standard Lyca camera mount, commonly used in aerial surveying and photogrammetric missions, and to operate at typical aircraft cruise altitudes between 8 and $12 \mathrm{~km}(25,000$ to $40,000 \mathrm{ft})$. The key characteristics of the IIP prototype instrument are listed in Table 3.

\begin{tabular}{|l|l|}
\hline Transmitted Laser Pulse Energy & $10 \mu \mathrm{J}$ \\
\hline Operating Wavelength & $532 \mathrm{~nm}$ \\
\hline Laser Fire Rate & $10 \mathrm{kHz}$ \\
\hline Transmitter Full Beam Divergence & $50 \mu \mathrm{rad}$ (FWHM) \\
\hline Aircraft Altitude & 8 to $12 \mathrm{~km}$ (cruise) \\
\hline Ground Spot Diameter & 40 to $60 \mathrm{~cm}$ (depending on altitude) \\
\hline Receive Telescope Diameter & $14 \mathrm{~cm}(87 \%$ aperture utilization) \\
\hline Detector QE & $12 \%(1,4$, or 16 pixels) \\
\hline Detection Threshold & 1 pe (photon-counting) \\
\hline Spectral Filter & $0.3 \mathrm{~nm}$ FWHM \\
\hline Receiver FOV & $140 \mu \mathrm{rad}$ (FWHM) \\
\hline Range Resolution & $5 \mathrm{~cm}$ (detector limited) \\
\hline Scan Frequency & $20 \mathrm{~Hz}$ \\
\hline Circular Scan (rotating optical wedge) & 300 to $500 \mathrm{~m}$ radius (depending on altitude) \\
\hline Effective Power-Aperture Product & 0.0013 W-m ${ }^{2}$ \\
\hline
\end{tabular}

Table 3: Nominal Operating Parameters of the NASA IIP Multikilohertz Microlaser Altimeter

\subsection{REPETITION RATE CONSIDERATIONS}

The airborne microaltimeter is designed to operate at $10 \mathrm{kHz}$ single photon sampling rates from aircraft cruise altitudes between 8 and $12 \mathrm{~km}(25,000$ to $40,000 \mathrm{ft})$. While other airborne laser altimeters [16] have operated successfully at these very high rates and have provided superb topographic maps, they typically use laser energies at least an order of magnitude higher and 
operate at very low altitudes ( 1 to $2 \mathrm{~km}$ ) where special FAA flight clearances are required. Thus, our choice of a $10 \mathrm{kHz}$ fire rate was driven by several factors:

1. We wanted to match the return rates of the best conventional altimeters but with a lower power-aperture product instrument operating at higher altitudes in order to demonstrate the technical and economic advantages of the microaltimeter approach.

2. The $10 \mathrm{kHz}$ rate allows system demonstrations to be carried out at aircraft cruise altitudes as high as $15 \mathrm{~km}(50,000 \mathrm{ft})$ without having to contend with multiple pulses in flight; this simplifies the receiver design.

3. It appeared to be a comfortable rate for modern data acquisition systems and would be compatible with the projected near term capabilities of space-qualified CPU's or Programmable Array Logic (PAL) processors.

The single pulse energy of $10 \mu \mathrm{J}$ and $14 \mathrm{~cm}$ telescope aperture provide a mean signal strength of about 1.5 pe per laser fire off a surface having a low reflectance on the order of 0.1 and a mean slope of approximately $45^{\circ}$. This results in a $76 \%$ probability of detection for the aforementioned reference slope and approaches $96 \%$ sampling for level terrain with a nominal soil reflectance of .15 so that we can achieve the nominal $10 \mathrm{kHz}$ surface sampling rate. As illustrated in Figure 1, a somewhat more efficient approach for the same power-aperture product would be to operate a 1 $\mu \mathrm{J}$ laser at $100 \mathrm{kHz}$ (for a return rate of about 0.1 pe per laser fire) and accept a roughly $90 \%$ data dropout, but this would have unnecessarily complicated the aircraft receiver and data acquisition by forcing it to track multiple pulses in flight at the much higher $100 \mathrm{kHz}$ rate. The latter issue will be addressed in our later discussion of multi-kHz spaceborne systems (Section 4) where the tracking of multiple pulses in flight is inevitable.

\subsection{INSTRUMENT OVERVIEW}

A block diagram of the NASA IIP instrument is presented in Figure 5. The system computer enables the firing of the passively Q-switched, frequency-doubled $\mathrm{Nd}$ :YAG microlaser transmitter and controls the repetition rate by adjusting the DC current to a $2.5 \mathrm{~W} \mathrm{CW}$ laser diode pump array. The diode pump light travels to the laser via an optical fiber having an emitting diameter of $100 \mathrm{~mm}$ and a Numerical Aperture (NA) of 0.22 and is refocused into the few $\mathrm{mm}$ long monolithic microchip by a short focal length lens. The microchip consists of a few $\mathrm{mm}$ long segment of Nd:YAG (the gain medium) diffusion-bonded to, or grown onto, a thin crystal of $\mathrm{Cr}^{4+}$-doped YAG, which acts as a passive saturable absorber $\mathrm{Q}$-switch and produces subnanosecond pulses. The matching indices of refraction and diffused nature of the interface results in low internal optical loss. The monolithic laser resonator is formed by polishing opposite sides of the microchip optically flat and parallel and applying reflective coatings [Zayhowski, 1998]. The resulting microlaser, which is only a few $\mathrm{mm}$ in length, produces a train of low energy $(\sim 10$ $\mu \mathrm{J})$, sub-nanosecond pulses at multi-kHz rates with no active switching components and, because of the monolithic structure, can never go out of alignment.

A small pick-off mirror injects the transmitter beam, following some initial divergence, into the central $5 \mathrm{~cm}$ of the common transmit/receive telescope, which uses a $14 \mathrm{~cm}$ off-axis parabola as the telescope primary mirror. Optical leakage from the outgoing laser pulse is sampled by a fast photodiode, which time-tags the start pulse with a Berkeley Nucleonics Corporation (BNC) Time-to-Digital Converter (TDC) and starts the range gate generator (BNC Model B940). The TEM $_{00}$ spatial mode quality $\left(M^{2}<1.3\right)$ of the end-pumped microlaser allows an extremely narrow transmit beam $(\sim 10$ arcsec) to be generated with a correspondingly small receiver FOV, which in turn reduces the solar noise count rate. The laser spot diameter on the ground varies between 40 and $60 \mathrm{~cm}$, depending linearly on aircraft altitude. The receiver utilizes the remaining $87 \%$ of the 
available aperture that is not blocked by the injection mirror to gather photons reflected from the surface. Stray light rejection within the receive telescope and interface optics severely restricts the number of off-axis photons reaching the stop detector. Internal gating of the detector and range receiver also helps prevent saturation of the detector resulting from laser backscattering within the instrument or aircraft.

The retuming photons pass through spectral $(0.3 \mathrm{~nm})$ and spatial $(\sim 140 \mu \mathrm{rad})$ filters and are imaged onto a 2x2 element Hamamatsu Model R5900U metal channel dynode Photo-Multiplier Tube (PMT). Because the primary timing unit, the BNC Model B945 TDC, can accommodate up to 16 stop events (one in each of 16 channels), the receiver design permits 4 stop events to occur within the range gate for each of the 4 detector elements. Provisions have also been made in the design to incorporate either a single element Model R7400U ( 1 channel, 16 stops) or a $4 \times 4$ Model H6568 PMT (16 channels, 1 stop per pixel) as alternate detectors. Use of pixellated photomultipliers permit a crude, but inexpensive, demonstration of the potential advantages of "quasi-imaging" or "point-to-point ranging". For example, at $12 \mathrm{~km}$ altitude where the ground beam diameter is about $60 \mathrm{~cm}$, the $4 \times 4$ array allows the source of the received photon to be resolved within a roughly $15 \mathrm{~cm} \times 15 \mathrm{~cm}$ cell on the ground. In a $300 \mathrm{~km}$ Mars orbit, the roughly $15 \mathrm{~m}$ diameter beam would be dissected into $4 \times 4$ meter cells by a $4 \times 4$ array leading to a higher resolution ground "image". Of course, improved spatial resolution could be achieved either through higher pixellation or the use of imaging photomultipliers as discussed previously in the Introduction. It is worthwhile to note that, to the extent that the signal and noise generation are both reasonably uniform within the receiver FOV, splitting the receiver FOV into individual pixels has no impact on the instrument contrast since the expected signal count and noise count per pixel are reduced equally.

Each detector pixel output is input to a high speed amplifier/constant fraction discriminator (AMP/CFD) module (Phillips Scientific Model 6908). The CFD provides a fast timing pulse to both a "coarse" and a "fine" range receiver. The function of the coarse receiver is to simultaneously capture all of the "soft" scattering surface returns (e.g. clouds, boundary or fog layers) as well as the ground retum and to provide an initial estimate of where the ground and near-ground scatterers (e.g. tree canopies and sub-canopies or man-made structures) are located so that the range gate on the fine receiver can be substantially reduced and optimally placed in time to concentrate on these surface features.

The "coarse" receiver is an EG\&G Ortec Turbo- Multi-Channel Scalar (MCS) which bins all of the photons received over virtually the entire fire interval and creates a histogram of photon stop events. The best coarse range resolution of $75 \mathrm{~cm}$ is determined by the minimum MCS bin size of $5 \mathrm{nsec}$. Since this level of range accuracy is more than adequate for clouds or other meteorological layers and there is similarly no need for high spatial resolution in the transverse dimension, the "stop" outputs of all the pixels are ORed together at the input to the MCS.

The "fine" range receiver time-of-flight (TOF) measurement is determined by differencing the time tags of the laser fire ("start") event and the photon return ("stop") events in the TDC. Although the TDC has a timing resolution of $50 \mathrm{psec}(7.5 \mathrm{~mm})$, the range resolution of the fine receiver is limited by the detector impulse response to about $5 \mathrm{~cm}$ RMS. Furthermore, since the TDC is capable of measuring only one stop event per channel, the serial "stop" pulses (up to a maximum of four) from each of the four pixels must first be separated into parallel lines by a 4 channel Serial-to-Parallel Converter (SPC). The SPC output fans out to the 16 channels of the TDC, thereby preserving pixel identification. 
In order of decreasing duration, individual gating pulses are applied to the PMT, the MCS, and the TDC respectively by the multi-channel range gate generator as in Figure 4 . In initial flights of the prototype instrument, all photon "times-of-arrival" within the range gate, relative to the start pulse, will be measured by the "fine" range receiver and recorded on the system hard disk for later analysis. However, our data extraction algorithms will be running in parallel to validate them in preparation for later flights, where software algorithms will identify the signal photons in flight and strip away most of the noise photons in near real-time.

There are three computers used in the flight system: the Ranging System Operator Interface (RGUI), the Ranging System Data Collector (RDATA), and the Navigational and Camera Control (NAV). A common timing reference is input to all three computers in the form of the current laser shot counter, which allows post-flight coordination of data recorded on separate computers.

The Ranging System Operator Interface is a $500 \mathrm{MHz}$ Pentium III processor running Windows NT 4.0. The system has 128 Mbytes of memory and two 13 Gbyte IDE Ultra ATA hard drives. One of the drives is used for system and program files; the other is mounted in a receiving frame for easy removal and will be used to record the flight data. The operator display is an LCD 20" rack mounted monitor with a pixel resolution of $1280 \times 1024$. The RGUI computer is responsible for operator interface, data logging, signal processing, and control of the EG\&G Turbo-MCS Histogrammer. In addition this computer picks up a small data set from the NAV computer at 1 $\mathrm{Hz}$ using internet file sharing, and it reads 720 Kbytes of shared memory data from the RDATA computer at $1 \mathrm{~Hz}$. Signal processing in RGUI provides visual feedback to the operator and can be used to provide control of the range window. All of the data is logged to the removable hard disk once per second. The amount of data recorded is approximately 3 Gigabytes per hour.

The basis of the RGUI software is NTGSE, which is a software package originally developed at GSFC for other missions. NTGSE has been modified for our application and has allowed a quicker software development cycle.

The Ranging Data Collector (RDATA) is a $233 \mathrm{Mhz}$ Pentium processor running DOS 6.22. The processor is an embedded module in a National Instruments VXI crate, which also contains a MXI interface to the RGUI computer (with 32Mbytes of shared memory), a digital I/O module (National Instruments DIO-128), and two Berkeley Nucleonics modules for ranging interface: the B940 digital delay generator (DDG) and the B945 16-channel time to digital converter (TDC). The embedded CPU contains 32 Mbytes of memory, a 4 Gbyte hard disk, as well as ethernet, SCSI, serial and parallel interfaces. RDATA uses an interrupt from the TDC module at the end of each range gate to trigger the reading of the laser fire time, the 16 range returns, the scan angle, and the shot counter. Interrupts are generated at the laser fire rate of $10 \mathrm{kHz}$. Data is placed in a shared memory buffer for the RGUI computer to pick up when it can. The shared memory buffer is a circular buffer and currently holds 5 seconds worth of data. Commands from the RGUI to the RDATA computer are also sent across shared memory. These include the range delay and the size of the range window, and are picked up by RDATA at $20 \mathrm{~Hz}$

The instrument will be flown with and without a simple scanner in order to demonstrate both contiguous 2D linear mapping of the underlying terrain as well as the 3D mapping of large swaths, extending over several hundred meters in the transverse direction, during a single pass of the aircraft. Because of the small size of the microaltimeter transmit/receive optics, an inexpensive optical wedge roughly $15 \mathrm{~cm}$ in diameter can be rotated at $20 \mathrm{~Hz}$ to superimpose a circular scan on the linear flight path motion. The rotating wedge, which deviates the beam from 
nadir by approximately 1 degree, simultaneously deflects both the transmitter beam and narrow receiver FOV while maintaining a common boresight.

\subsection{POST-FLIGHT DATA PROCESSING}

In order to generate an accurate topographic map, it is essential for any laser altimeter that we know both the instantaneous position and attitude of the instrument on each laser fire in addition to the pulse time-of-flight and any fixed timing biases within the instrument. The latter bias can be determined by ranging to a target whose distance from the instrument reference point is well known. For the quasi-imaging and scanning microaltimeter, one must also make corrections based on the instantaneous off-nadir angle of the scanner and the $\mathrm{X}-\mathrm{Y}$ coordinate (or pixel) which recorded the photon event.

Multiple GPS geodetic receivers will be used (one in the aircraft and one or more on the ground) to provide post-flight dynamic differential positioning at the few decimeter level. The fixed offset vector between the GPS antenna phase center and the altimeter reference point in the aircraft reference frame will be measured and corrected by three-axis attitude data to accurately locate the altimeter reference point in the Earth reference frame during flight. Instantaneous instrument attitude will be provided by a three-axis fiber-optic gyro mounted directly on the altimeter optical bench and calibrated pre-flight. To counter or monitor gyro drift errors, independent updates of attitude will be obtained in-flight by (1) a digital compass (for heading) supplemented by two collocated and orthogonal bench-mounted inclinometers and (2) repeatedly over-flying a set of four "ground stars", whose relative positions have been precisely located at the few $\mathrm{mm}$ level using geodetic GPS receivers. These "ground stars" will be located near the center of the interrogated region to accommodate frequent overflights and will be arranged in a square or diamond pattern a couple of $\mathrm{km}$ on a side. Thus, when the aircraft is near the center of the pattern, they can all be viewed simultaneously by the wide FOV camera attached to the altimeter instrument, providing adequate angular resolution for updating the aircraft attitude at the few arcsecond level.

One candidate approach to generating the ground stars is to use small battery-powered Light Emitting Diode (LED) arrays, which are pulsed by the lpps output of a GPS Timing Receiver. The onboard digital camera, boresighted with the range receiver and synchronized with the wideangle ground star emission by the lpps output of the onboard GPS receiver, then records the spectrally narrow images of the ground stars through a bandpass filter. The deviation of the ground stars from their predicted positions in the camera FOV, based on the few decimeter accuracy aircraft positioning, allows the instrument attitude to be updated post-flight with few arcsecond accuracy.

The instantaneous angular position of the scanning wedge is obtained by interpolating between multiple sensors which monitor the passage of a particular point on the wedge by producing an output pulse which is time-tagged and recorded in the data file along with the ranging data. This was viewed as an adequate and less expensive and data intensive approach than using encoders due to the fairly constant wedge velocity between monitoring points. As mentioned previously, the detector pixel producing a given photon event is recorded by the "fine" range receiver and can be used to correct the measured elevation for the transverse location of the photon source within the ground spot.

To summarize, a "start" pulse and potentially one or more "stop" pulses (noise and/or signal counts) will be recorded, for each laser fire, by the coarse and fine receivers, both of which can be viewed as correlation range receivers but with vastly different range gates and resolutions. The 
post-detection Poisson filtering techniques described in Section 2.5 will identify which cells in the coarse and fine receivers are most likely to contain signal counts. The photon TOFs for the echos selected as signal must then be subtracted from the aircraft altitude and corrected for pitch, roll, and yaw in order to determine the terrain heights. Additional corrections for the imposed off-nadir pointing due to the rotating scanner must also be made. Approximate X-Y locations, as determined by the pixellated or imaging detector, further allow more accurate placement of the measured terrain heights in the transverse dimension.

The final analysis data product will be a three dimensional plot (and corresponding data files) of the terrain or sea heights for the scanned swath $(< \pm 200 \mathrm{~m})$ beneath the flight path. Early calibration flights will concentrate on target areas which have been accurately mapped by conventional altimeters or other techniques. Comparisons between existing Digital Elevation Models and our own terrain measurements will be produced, giving point-to-point height differences and an overall measure of terrain difference

Follow-on science flights will investigate instrument performance over a variety of terrain Barren terrain represents the simplest target for the microaltimeter and will be useful in evaluating and optimizing the technique for future planetary topographic mapping missions. Highly vegetated surfaces are much more complex but will determine whether or not the microaltimeter can successfully recover canopy and sub-canopy heights via statistical interpretation of the high repetition rate data. Towns and cities will test the ability of the instrument and data processing algorithms to record and adapt to frequent and large elevation changes and assess its value as an aerial surveying tool. Finally, overflights of beach areas will evaluate its capability for monitoring beach erosion and wave heights and for performing shallow water bathymetry at single photon levels.

\section{OF THE AIRBORNE MICROALTIMETER PERFORMANCE AT 12 KM ALTITUDE}

Our analysis of the airborne microaltimeter is summarized by the six plots in Figure 6. Each graph contains three curves, labeled by the assumed value of $\rho$ for a wavelength of $532 \mathrm{~nm}$, e.g. $\rho=1$ (snow, thick clouds), 0.15 (soil or dense dry vegetation), 0.1 (dense green vegetation). A slope of 45 degrees was assumed which reduces the mean signal by a factor of 0.707 and results in a single shot retum waveform of about $4 \mathrm{nsec}$. The noise models derived in Appendix A indicate that 2 to 4 noise counts per laser fire are expected to occur over most terrain within a 4 microsecond gate which is well within the sixteen stop limit of the receiver. Over highly reflective clouds or snow, this can grow to 11 counts per fire. A receiver dead-time of $10 \mathrm{nsec}$ was estimated for the aircraft hardware so that Eqs. (28) through (30) apply. A nominal 4 microsecond timing gate was assumed, which corresponds to a "fine" receiver range window of $600 \mathrm{~m}$. The latter value would accommodate detailed topographic mapping of the tallest forests (Giant Sequoias grow to a height of almost $100 \mathrm{~m}$ ) and the tallest skyscrapers in major cities (the top of the Sears Tower in Chicago is $442 \mathrm{~m}$ above street level). For simplicity, it is further assumed that the microaltimeter is operating in an unscanned (2D) mode with a single element detector.

Figure 6a plots the maximum surface sampling rate, $f_{\max }$, versus the power-aperture product for an altimeter operating at the maximum cruise altitude of $12 \mathrm{~km}$ as computed using (4) and the instrument parameters in Table 3. It can be seen from the figure that the minimum power-aperture product required to achieve $10 \mathrm{kHz}$ surface sampling from the worst case surface is $1 \times 10^{-3}$ Watt$\mathrm{m}^{2}$. This is to be compared to our actual instrument value of $1.34 \times 10^{-3} \mathrm{~W}-\mathrm{m}^{2}$, which is chosen about $34 \%$ larger in order to have a high probability of detection on each laser fire with a nominal 
mean signal strength of 1 pe per laser fire (see Figure 1) and to avoid the need to operate the system at $100 \mathrm{kHz}$ rates as described previously in section 3.2 .

Figure $6 \mathrm{~b}$ plots the minimum cell contrast as a function of the number of laser fires per frame (frame interval). The cell contrast decreases with increasing frame interval because of the need to widen the range bin according to Eq. (23a). Over the range of 1 to 100 laser fires per frame, which is common to Figures 5(b) though 5(f), the frame interval increases from 0.1 to $10 \mathrm{msec}$, the range bin width varies from 4 to $10 \mathrm{nsec}$, the corresponding number of range bins decreases from 1000 to about 400 , and the ground distance covered by the aircraft at a velocity of $100 \mathrm{~m} / \mathrm{sec}$ varies from $1 \mathrm{~cm}$ to $1 \mathrm{~m}$.

Figure $6 \mathrm{c}$ plots the optimum frame threshold as a function of the number of laser fires per frame as computed from (19) and rounded off to the next highest integer.

In Figures 6d through $f$, we plot the Differential Cell Count (normalized to $M$ ), the probability of correctly identifying the signal cell, and the mean number of falsely identified cells per frame respectively as a function of the frame interval. We note that the Normalized Differential Cell Count approaches the ideal value of unity for 15 laser fires per frame or a frame interval of 1.5 msec. As expected, the probability of detecting the signal cell also approaches unity and the mean number of falsely acquired noise cells per frame falls well below unity for these longer frame durations. However, one should choose the minimum frame duration that gives acceptable results since ultimately the range bin can grow to encompass a large fraction of the range gate which limits the effectiveness of the selection algorithm as a real-time noise filter.

It should be noted that these analyses assume a worst case solar noise background as calculated in Section 2.4. The frame threshold and frame interval can be initially set based on a priori expectations and then recomputed in flight using (19) and actual measurements of the mean signal and noise counts per range cell. A smaller mean noise count will increase the contrast, which in turn reduces the threshold and the required frame interval. Adaptively narrowing the range gate over barren land or low vegetation to reduce the value of $N_{\text {bin }}$ in (19) has a similar effect.

Figure 7 shows the results of a Monte-Carlo simulation of the microaltimeter operating in nonscanning mode over a rapidly changing terrain devoid of any ground vegetation. The simulated surface, which includes a 50 meter cliff, is easily seen against the solar background noise in the coarse map of Figure 7a. In spite of the steep cliff, the selection algorithm was able to rapidly identify and reacquire the surface returns from the top of the cliff.

The expanded scale in Figure $7 \mathrm{~b}$ shows the actual roughness for a segment of the simulated surface in Figure $7 \mathrm{a}$ as well as the data points selected by the algorithm as surface returns (points surrounded by circles). The few noise counts falsely identified as signal in Figure $7 \mathrm{~b}$ happened to fall into the chosen signal cells and would be eliminated in iterative applications of the selection algorithm if one substitutes the bin size of the signal cell for the range gate and subdivides it into still smaller range bins on each iteration. 


\subsection{SPACEBORNE ALTIMETERS FOR PLANETARY TOPOGRAPHIC MAPPING}

\subsection{ADDITIONAL TECHNICAL CONSIDERATIONS FOR SPACEBORNE MISSIONS}

To simplify our discussion of spacebome microaltimeters, we will consider a planet other than Earth, whose surface topography is uniquely complicated by the presence of vegetation and manmade structures. We will also consider a non-scanning system with a single element detector. In scaling the microaltimeter from airborne to spaceborne platforms, we encounter the following technical issues:

1) The required power-aperture product for the altimeter scales as $R^{2}$ for a given surface sampling rate, but, if the receiver dead time is long compared to the single shot surface return waveform, the receive aperture will necessarily be limited by the need to keep noise counts within acceptable limits.

2) One must contend with multiple pulses in flight in order to achieve $\mathrm{kHz}$ or higher sampling rates from orbit.

3) Higher spacecraft ground velocities imply a faster rate of growth in the required range bin with increasing frame interval and modest surface slope. In addition, the roundtrip transit time to the surface is on the order of milliseconds which contributes to the uncertainty in the predictive range window and further increases range bin duration.

4) Prime power, size, and weight play a more significant role in spaceborne instrumentation, especially for interplanetary missions.

We will address each of these topics as we proceed with an analysis of a Mars topographic mission.

\subsection{ONE METHODOLOGY FOR DESIGNING A MULTI-KHZ MARS MICROALTIMETER}

Using our theoretical results from Section 2, we will now illustrate one methodology for designing an optimized multi-kHz microaltimeter to rapidly map the surface of Mars. The design process begins by: (1) selecting the minimum sampling rate one wishes to achieve over a nominal worst case surface (i.e., maximum median slope and minimum reflectance); (2) minimizing the instrument optical losses; (3) minimizing the noise background via spatial and spectral filtering and utilizing a correspondingly low transmitter beam divergence; and (4) selecting a high quantum efficiency detector.

A typical altitude for a Martian satellite (e.g., MOLA) is $300 \mathrm{kM}$ with a corresponding ground velocity slightly greater than $3 \mathrm{~km} / \mathrm{sec}$. Thus, if we wanted to sample the worst case surface every meter along-track, we would set $f_{s}=3 \mathrm{kHz}$. We note from (3) that, in photon counting mode, the sampling rate, $f_{s}$, is usually less than the laser fire rate, $f_{q s}$, since the probability of detecting a signal photon is less than unity unless $n_{s}>>1$.

Assuming a $50 \mu \mathrm{rad}$ full beam divergence, which is easily achieved with a transmit aperture of one to two inches and the TEM00 mode quality of the microlaser source [Zayhowski, 1998], the beam diameter on the ground is $15 \mathrm{~m}$. Thus, the mean spacing between subsequent spots on the ground is about $7 \%$ of the beam diameter and the retums are therefore highly contiguous. We will

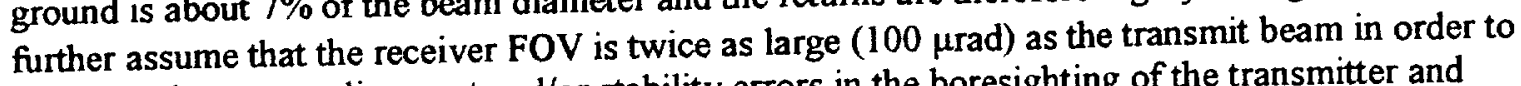
accommodate some alignment and/or stability errors in the boresighting of the transmitter and receiver. 
The median slope measured by MOLA, on $300 \mathrm{~m}$ beam centers along-track and averaged over 35 $\mathrm{km}$ baselines, as analyzed by MIT rarely exceeded 5 degrees and the legend on their global map shows a maximum plotted value of about 23 degrees [Aharonson, 2000], which we will adopt as $\sigma_{\max }$. We will also assume that the nominal reflectance of soil on Mars is comparable to that on Earth, i.e. about $15 \%$ at $532 \mathrm{~nm}$. Locally, the slopes and reflectances may vary about these values leading to both increases or decreases in the photon return rate from the surface, but our autotracking algorithm is capable of adjusting to these variances.

Over high reflectance surfaces (if any), the mean signal photon counts will increase, and the microaltimeter sampling rate will approach a maximum determined by the laser fire rate. However, because of the receiver dead time effects discussed in Section 2.8, we must include an optical attenuator in our receiver design in order to reduce the mean noise count per cell and maintain the system contrast at an acceptable level by mimicking a lower reflectance surface. Without the attenuator, a sufficiently bright surface would be indistinguishable from the solar background.

The GLAS project plans to use a space-qualified photon-counting detector with a quantum efficiency greater than $50 \%$ at $532 \mathrm{~nm}$ [Abshire et al, 2000]. The SPAD detector is actively quenched with a dead time of $50 \mathrm{nsec}$. The principal optical loss element in the receiver is the narrowband spatial filter. Spectral bandpass filters with good optical throughput $(>50 \%)$ at 532 $\mathrm{nm}$ are generally limited to a spectral bandwidth of $\Delta \lambda=3 \mathrm{~A}^{\circ}$ FWHM. We will adopt this value for the purposes of this analysis and assume a total receiver optical throughput efficiencies of $\eta_{r}=$ 0.4 . If necessary, spectral filtering can be further enhanced, via a thermally tuned etalon filter, to as little as $0.3 \mathrm{~A}^{\circ}$ with an additional $15 \%$ loss in throughput as in the GLAS instrument [Abshire et al, 2000].

\begin{tabular}{|l|l|}
\hline \multicolumn{2}{|c|}{ Instrument Characteristics } \\
\hline Wavelength, $\lambda$ & $532 \mathrm{~nm}$ \\
\hline Detector Quantum Efficiency, $\eta_{q}$ & 0.5 \\
\hline Detector Pixels, $N_{p}$ & 1 \\
\hline Receiver Optical Efficiency, $\eta_{r}$ & 0.4 \\
\hline Transmitter Full-Angle Divergence, $2 \theta_{t}$ & $50 \mu \mathrm{rad}$ \\
\hline Receiver Full Angle FOV, $2 \theta_{r}$ & $100 \mu \mathrm{rad}$ \\
\hline Spectral Filter Bandwidth, $\Delta \lambda$ & $3 \mathrm{~A}^{\circ}$ \\
\hline Receiver Dead-Time, $\tau_{d}$ & $50 \mathrm{nsec}$ \\
\hline Noise count rate per unit receive area, $\beta$ & $5 \mathrm{x} 10^{7} / \mathrm{m}^{2}-\mathrm{sec}$ \\
\hline Minimum sample rate, $f_{s}$ & $3 \mathrm{kHz}$ \\
\hline \multicolumn{2}{|c|}{ Spacecraft Characteristics } \\
\hline Altitude, $R$ & $300 \mathrm{~km}$ \\
\hline Ground Velocity, $v_{s}^{\text {eff }}$ & $3.13 \mathrm{~km} / \mathrm{sec}$ \\
\hline \multicolumn{2}{|c|}{ Surface Characteristics } \\
\hline Reflectance, $\rho$ & 0.15 \\
\hline Maximum Median Slope, $\sigma_{\max }$ & $23^{\circ}$ \\
\hline \multicolumn{2}{|}{ Atmospheric Characteristics } \\
\hline One-Way Transmission at Nadir & 0.90 \\
\hline
\end{tabular}

Table 4: Summary of parameters used in Mars microaltimeter analysis. 
Substituting the aforementioned values, which are summarized in Table 4, into (4) and solving for the minimum power-aperture product yields a value $P A_{m n}=0.017 \mathrm{~W}-\mathrm{m}^{2}$. For night only operations, one can arbitrarily trade off receive aperture and laser power to achieve the necessary product. However, we will now demonstrate that, in the presence of solar noise, this minimum power aperture product, which from (3) implies a mean signal count per laser fire on the order of $0.1 \mathrm{pe}$, is not necessarily the optimum choice. In order to reduce the mean noise count per range bin well below 0.1 pe and maintain adequate cell contrast, the receive aperture must be kept relatively small, placing a greater burden on the laser transmitter power to achieve the required power-aperture product. In all potential instrument configurations, the receive aperture requirements are fairly modest $(10$ to $20 \mathrm{~cm}$ ), and one may wish to choose an instrument configuration which places the fewest demands on the laser.

Since Mars lies a mean distance of $1.524 \mathrm{AU}$ from the Sun, the spectral irradiance, $N_{\lambda}{ }^{0}$ impinging on the planet's atmosphere is reduced by a factor of 2.32 relative to Earth to a value $0.086 \mathrm{~W} / \mathrm{m}^{2}$ $A^{\circ}$ at $532 \mathrm{~nm}$. Furthermore, since Mars' thin atmosphere is expected to be more highly transmissive than Earth's, the atmospheric scattering component of the noise is reduced relative to the surface component. For the purposes of our analysis, we will assume an atmospheric transmission of $90 \%$. From (A8) and (A11) in Appendix A, we calculate a worst case solar count rate per unit receive area of $4.2 \times 10^{7} / \mathrm{m}^{2}$-sec from the Martian surface and $8.2 \times 10^{6} / \mathrm{m}^{2}$-sec from the intervening atmosphere. Thus, the total worst case solar noise count rate is $\beta=5.0 \times 10^{7} / \mathrm{m}^{2}$ sec with $84 \%$ due to surface scattering. If we assume a typical receiver dead-time, $\tau_{d}$, of $50 \mathrm{nsec}$ in (30), we obtain an upper limit on the receive aperture of $A_{\max }=1 / \beta \tau_{d}=0.4 \mathrm{~m}^{2}$, corresponding to a maximum receiver diameter of $71 \mathrm{~cm}$ in the low contrast limit.

We demonstrated in Section 2.5 that different combinations of contrast and frame time can provide roughly the same ability to distinguish signal from noise. The required signal count per frame, $N_{s}$, increases with decreasing signal cell contrast as demonstrated in Figure 4 . The frame duration is given by the formula $\tau_{f}=N_{s} / f_{s}$. The corresponding range bin can be computed from (23) with $\gamma=1.77 \times 10^{-5}$. The results are summarized in the following table:

\begin{tabular}{|c|c|c|c|}
\hline $\begin{array}{c}\text { Contrast, } \\
\mathbf{C}\end{array}$ & $\begin{array}{c}\text { Signal Counts per Frame, } \mathbf{N}_{\mathbf{s}} \\
\text { Ground Track per Frame }(\mathbf{m})\end{array}$ & $\begin{array}{c}\text { Frame Duration, } \\
\boldsymbol{\tau}_{\boldsymbol{f}} \text { (msec) }\end{array}$ & $\begin{array}{c}\text { Range Bin, } \\
\boldsymbol{\tau}_{\mathbf{b}} \text { (nsec) }\end{array}$ \\
\hline 2 & 80 & 27 & 515 \\
\hline 3 & 50 & 17 & 338 \\
\hline 5 & 35 & 12 & 249 \\
\hline 10 & 25 & 8 & 190 \\
\hline
\end{tabular}

Table 5: Combinations of contrast and mean signal counts per frame which provide roughly the same ability to discriminate signal from noise. Because for $f_{3}=3 \mathrm{kHz}$ we make on average one range measurement for every meter of ground travel, the ground track covered per frame is roughly equal to $N_{s}$ in meters. The corresponding frame durations and range bins for the postulated Mars mission are computed using various formulas cited in the text.

We now let the mean signal count per laser fire, $n_{s}$, vary between 0.1 and 10 pe and compute in succession the following quantities:

Single-shot probability of detection: $P_{d}=1-\exp \left(-n_{s}\right)$

Laser Fire Rate: $f_{Q S}=\frac{f_{s}}{P_{d}}$ from (3) 
Receiver Area: $A_{r}=\frac{1}{\beta\left[\frac{C-1}{P_{d}} \tau_{b}+\tau_{d}\right]}$ from (30)

Transmitted Single Pulse Energy: $E_{t}=\frac{n_{s} R^{2}}{C_{a} A_{r}}$ from (1)

Transmitted Laser Power: $P_{t}=f_{Q S} E_{t}$

Power-Aperture Product: $P_{t} A_{r}=\frac{n_{s}}{1-e^{-n_{s}}} \frac{f_{s} R^{2}}{C_{a}}$

The results are plotted in Figure 8 as a function of the mean signal count per laser fire over a range $0.1 \mathrm{pe}<n_{s}<10 \mathrm{pe}$. In, Figure $8 \mathrm{a}$, the single shot probability of detection is greater than $98 \%$ for $n_{s}>4$. Figure $8 \mathrm{~b}$ illustrates the fact that, at low mean signal strengths on the order of 0.1 pe, the altimeter must be operated at $30 \mathrm{kHz}$ in order to achieve the $3 \mathrm{kHz}$ sampling rate and compensate for the low probability of detection. The required laser fire rate falls asymptotically to $3 \mathrm{kHz}$ as the probability of detection asymptotically approaches unity with an increased mean signal count. In (c) through (e), the four curves are labeled by the assumed value for the contrast, $C$, with the corresponding value of the mean signal count per frame, $N_{s}$, given by Table 5 . In Figure 8c, we use (30) to demonstrate that a stronger mean signal count allows the receiver diameter to grow until the mean noise count per bin per fire gets close to the limiting value, $n_{b}=$ $1 /(C-1)$. This results from the fact that the system dead-time allows the detection of only one signal photoelectron per laser fire as discussed in Appendix B. Since the receiver area cannot grow further once this limit is reached, the transmitted pulse energy must increase to achieve still higher mean signal counts as in Figure 8d. Figure 8e provides the interesting and useful result that the required average laser power reaches a minimum for all contrasts at a mean signal count of about 1.24 pe. As the desired contrast increases, however, the required laser power also increases, again due to dead-time effects which constrain the receive aperture. Finally, the Power-Aperture product is plotted in Figure 8f, and, as expected, all four contrast curves overlap perfectly. As expected from the theoretical results in Section 2.1, the power-aperture product required to achieve a given sampling rate increases monotonically with increasing $n_{s}$.

Returning to the point of minimum transmitter power in Figure 8e, we note that a transmitted laser power of only one watt, coupled with a very modest $18 \mathrm{~cm}$ diameter receive telescope, would allow us to do nominal 3 to $4.3 \mathrm{kHz}$ ranging to the Martian surface from a $300 \mathrm{~km}$ orbit, provided we accept a minimum signal contrast of 2 . The laser would operate at roughly $4.3 \mathrm{kHz}$ with a single pulse transmitted energy of only $230 \mu \mathrm{J}$. Choosing a higher signal contrast of 10 would allow use of a smaller $10 \mathrm{~cm}$ telescope, with perhaps a correspondingly smaller and lighter scanning mechanism (if relevant), but it also would require a larger and heavier 3 watt laser (700 $\mu \mathrm{J} @ 4.3 \mathrm{kHz}$ ) with approximately three times the onboard power requirements. As demonstrated in Figure 8e, the power-aperture products would be identical for the two systems $\left(0.024 \mathrm{~W}-\mathrm{m}^{2}\right)$, but the lower contrast system would place less of the signal strength burden on the laser. This would normally make it the system of choice since, by conventional spaceborne altimeter standards, the telescope size and weight is very modest in either case. The principal advantage of high contrast systems is that fewer noise events are retained by the autotracking algorithm in real time, but, as discussed in Section 2.5, in-flight post-processing of the data can easily remove 
these. As a point of reference, the power-aperture product for the MOLA altimeter is almost 4 times larger, i.e. $\mathrm{PA}_{\mathrm{MOLA}}=0.088 \mathrm{~W}-\mathrm{m}^{2}$, yet the sampling rate is 300 times smaller $(10 \mathrm{~Hz}$ vs 3 $\mathrm{kHz}$ ) for the nominal "worst case" surface.

To summarize, we argued in Section 2.1 that operating the altimeter at low mean signal levels $\left(n_{s}\right.$ $\leq 0.1)$ allowed us to maximize the sampling rate for a given power-aperture product. In the absence of background noise, we are free to arbitrarily choose the transmitted power and receive aperture which yield this product. In the presence of noise, however, the receive aperture must be severely limited in order to maintain the necessary signal contrast (i.e. $n_{b}<n_{s}<0.1$ ), which in turn places more of the signal burden on the laser transmitter. By operating at a higher signal level slightly greater than 1 pe (and hence a somewhat higher power-aperture product), we can increase the size of the receive aperture and minimize the required transmitter power.

\subsection{TIMING OF MULTIPLE PULSES IN FLIGHT}

In the $10 \mathrm{kHz}$ airborne system discussed in Section 3, only one pulse is in flight at a given time for aircraft altitudes below $15 \mathrm{~km}$. Thus, a simple event timer, triggered by the outgoing pulse, is sufficient for the range measurement. For a $4 \mathrm{kHz}$ microaltimeter operating from a circular orbit $300 \mathrm{~km}$ above Mars, however, there are approximately eight pulses simultaneously in flight, separated by roughly $75 \mathrm{~km}$.

The problem of designing a correlation range receiver (CRR) which handles multiple pulses in flight has been addressed previously in connection with NASA's developmental photon counting SLR2000 satellite laser ranging system, designed to operate with segmented anode photomultipliers at a $2 \mathrm{kHz}$ laser fire rate. An early version of the receiver [Degnan and McGarry, 1997 had a demonstrated RMS ranging resolution of $4 \mathrm{~mm}$ and was later improved to about 1 $\mathrm{mm}$ [Degnan, 1998]. Since such high range resolution is unwarranted for an altimeter designed to have few $\mathrm{cm}$ resolution, one can consider simpler and more power efficient alternatives for the timing of photon "events".

The range receiver is designed around a "sub-clock" which produces timing pulses at the chosen laser fire rate, $f_{\mathrm{QS}}$, and is derived through frequency division from the high rate output (typically $10 \mathrm{MHz}$ ) of the spacecraft clock/frequency standard. This "sub-clock" pulse can then be used to trigger both the microlaser transmitter pump diodes and a timing receiver, which is operated modulo the laser fire interval $(\sim 250 \mu \mathrm{sec}$ for our $4 \mathrm{kHz}$ Mars example). The pump diodes produce a rectangular optical pulse which pumps the Q-switched microlaser until laser threshold is reached, at which point an intense subnanosecond pulse is rapidly produced. The timing jitter between the laser pump trigger and the emission of the laser pulse can be large compared to the desired few hundred picosecond timing resolution. Thus, to achieve the necessary range accuracy, the temporal positions of the outgoing pulses relative to the sub-clock "tick" must be measured on each fire. The return photon from the Martian surface arrives 8 fire cycles later, and its time of arrival is again measured relative to the sub-clock timing pulse. By properly pairing the "start"

and "stop" events, accurately measuring their temporal positions relative to the sub-clock "ticks", and adding the appropriate number of intervening sub-clock cycles, an accurate time-of-flight measurement can be obtained [Degnan and McGarry, 1997]. Thus, each photon event can be labeled by: (1) the epoch time second on the spacecraft clock preceding the measurement; $(2)$ the laser fire interval within the following second $(0-4000$ for a $250 \mu \mathrm{sec}$ interval) during which the photons departed the instrument ("start event") or were received ("stop event"); and (3) the time interval between the sub-clock pulse and the photon event as measured by the modulo timer. 
Because of the large $75 \mathrm{~km}$ spacing between pulses, the number of intervening clock cycles can be discerned from a priori knowledge of the satellite altitude obtained from navigation data.

Furthermore, at $4 \mathrm{kHz}$, the $250 \mu \mathrm{sec}$ fire interval allows a range depth up to $37.5 \mathrm{~km}$ to be probed, which is long enough to easily accommodate the entire topographic range of the inner planets.

For example, we note that the highest point on Earth, Mt. Everest in Nepal, lies roughly $8.8 \mathrm{~km}$ above mean sea level. Thus, all surface and canopy returns (plus most low lying clouds of interest for radiation transfer studies) could be accommodated within a fixed range gate on the order of 60 $\mu \mathrm{sec}$ if one were to assume a fixed receiver range gate, a highly circular orbit, no terrain below sea level, and a truly spherical planet. In reality, because of the planet's oblateness, an altimeter in circular orbit about the Earth would require a slightly larger temporal window on the order of 75 $\mu$ sec to accommodate the global surface retums. Still, this global variation in surface topography represents only $33 \%$ of the 250 microsecond period between pulses. Extending the range window to span either the entire $20 \mathrm{~km}$ height of the Earth's atmosphere to include high cloud returns for radiation balance measurements or the highest feature on Mars, Olympus Mons at $21 \mathrm{~km}$ [Frey, 2000 ], causes a global spread up to $146 \mu \mathrm{sec}$ or $55 \%$ of the laser fire period. Furthermore, since the laser fire is triggered by the sub-clock ticks and the jitter in the pulse output is also a relatively small fraction of the fire period, the start and stop pulses can be temporally isolated from each other within the laser fire period via a fixed range gate generator and input to a single modulo timer to conserve prime power.

At laser fire rates greater than about $7.5 \mathrm{kHz}$, the range return intervals from different laser fires will begin to overlap in time at the receiver for probed range depths on the order of $20 \mathrm{~km}$, making data interpretation more difficult. To avoid this complication, higher repetition rate systems would need to reduce the range depth and concentrate on surface and/or near-surface retums (e.g. tree canopies and low lying cumulus clouds.

Finally, if the $250 \mu \mathrm{sec}$ laser fire interval for our Mars Microaltimeter is broken up into 500 range bins, as assumed in Figure 4, the resulting range bin duration of $500 \mathrm{nsec}$ is a good match for the $\mathrm{C}=2$ case in Table 5 , which required the least transmitter power and hence was the instrument configuration of choice.

\subsection{A POWER EFFICIENT ALL-DIGITAL TIMER}

As mentioned in the Introduction, conventional laser altimeters often make use of high speed time digitizers which record the waveform of the multi-photon returns. When compared to digital circuits, these analog devices tend to consume large amounts of prime power as and require the use of complex (and often fallible) algorithms to compute the range to complex surfaces. On the other hand, computing the time-of-flight of a single photon is a relatively unambiguous process and, combined with the quasi-imaging capabilities of pixellated detectors, permits greater spatial resolution in both the horizontal and vertical directions. Furthermore, the modest ranging resolution (few $\mathrm{cms}$ ) required by the altimeter allows one to consider a compact and powerefficient all-digital design for the modulo timer [Degnan et al, 1999].

A block diagram of the All-Digital Event Timer, with an integrated $6 \times 6$ micro-APD array, is shown in Figure 9. A $4 \mathrm{GHz}$ clock is derived from a voltage controlled crystal oscillator and is phase-locked to the $10 \mathrm{MHz}$ frequency standard. A 21 bit synchronous counter is used to time tag photon events from the 36 element array. An input signal conditioner is used to "latch" the incoming photon events to the nearest $4 \mathrm{GHz}$ clock pulse, providing a timing resolution of 250 psec. The latch stores the counter data to time the event. An array location data generator identifies which pixel of the array has been excited by the incoming photon. The pixel location 
information and the high precision timing information are then fed to a 64 bit FIFO (First In First out) buffer. The resulting FIFO output is a 64-bit word that contains the time of the occurrence of the event and the location of the excited pixel. We estimate that low voltage $(\leq 3 \mathrm{~V})$ integrated circuits (IC's) and differential signal (LVDS) drivers and receivers can reduce power consumption of the timer to less than 6 watts in a very small package occupying about 5 cubic inches.

\subsection{SUMMARY}

We have described a photon-counting laser altimeter whose advantages include: (1) a more efficient use of available laser photons providing up to two orders of magnitude greater surface sampling rates for a given power-aperture product; (2) a simultaneous two order of magnitude reduction in the volume, cost and weight of the telescope system; (3) the unique ability to spatially resolve the source of the surface retum in a photon counting mode through the use of pixellated or imaging detectors; and (4) improved vertical and transverse spatial resolution resulting from both (1) and (3). Furthermore, because of the significantly lower laser pulse energies, the microaltimeter is inherently more eyesafe to observers on the ground and less prone to the internal optical damage, which can terminate a space mission prematurely.

\section{ACKNOWLEDGEMENTS}

Funding for the Airborne Multi-kilohertz Microlaser Altimeter ("Microaltimeter") has been provided by NASA's Instrument Incubator Program. The author wishes to acknowledge the contributions of several members of the NASA Microaltimeter Team at the Goddard Space Flight Center who have collaborated in the instrument design described briefly in Section 3 of this paper. Ms. Jan McGarry has led the controller and software design effort and has modified an existing altimeter simulator to generate the simulation in Figure 7. She has been aided in the development of system software by Jenny Geiger and Peter Abel. Thomas Zagwodzki developed the engineering design of the dual range receiver for the airbome system, and Phillip Dabney leads the effort on aircraft navigation, attitude determination, and "ground star" calibration. Joseph Marzouk and Marcos Sirota of Sigma Research and Engineering Corporation (SREC) designed the optical bench, telescope, and interface optics. Charles Steggerda of Honeywell Technical Solutions Inc. is the lead engineer on the $4 \mathrm{GHZ}$ All-Digital Modulo Timer, and the prototype circuit board was developed under our Interplanetary Microlaser Transponder Program and was funded by the Goddard Director's Discretionary Fund.

\section{APPENDIX A: ALTIMETER NOISE MODEL}

\section{A.1 SOLAR SCATTERING FROM THE ATMOSPHERE}

Because the effective height of the atmosphere above sea level $\left(\mathrm{h}_{\mathrm{a}} \sim 20 \mathrm{Km}\right)$ is small relative to the mean radius of the Earth $\left(R_{E}=6378 \mathrm{Km}\right)$, we can represent the atmosphere by a stratified rectangular slab with the interrogated surface centered on the bottom face. The atmospheric extinction coefficient, $\mu(z)$, is assumed to be a function of the vertical height only and no horizontal gradients are assumed. The atmospheric transmission at nadir is then given by

$$
T_{0}=T\left(h_{s}, \infty\right)=\exp \left[-\int_{h_{s}}^{\infty} d z^{\prime} \mu\left(z^{\prime}\right)\right]
$$


where $h_{s}$ is the height of the surface above sea level, and we assume the altimeter is located well above the attenuating atmosphere.

The solar irradiance can be viewed as a plane wave incident on the top plane of the atmosphere at a solar zenith angle, $\theta_{\mathrm{g}}$. If we ignore the slight bending of the solar rays as they propagate through the vertically stratified atmosphere, the transmitted irradiance to a differential volume at an arbitrary height above sea level, $z$, is given by

$$
N_{\lambda}^{0} \exp \left(-\sec \theta_{s} \int_{z}^{\infty} d z^{\prime} \mu\left(z^{\prime}\right)\right)=N_{\lambda}^{0}[T(z, \infty)]^{\sec \theta_{s}}
$$

where $N_{\lambda}^{0}$ is the extraterrestrial solar irradiance impinging on the Earth's atmosphere which, at $532 \mathrm{~nm}$, has a value $N_{932}^{0}=0.2$ watts $/ \mathrm{m}^{2}-\mathrm{A}^{0}$.

The altimeter receiver has a solid angle field of view (FOV) given by

$$
\Omega_{r}=\pi \theta_{r}^{2}
$$

where $\theta_{\mathrm{r}}$ is the half angle of the far field cone viewed by the receiver. The scattered solar power collected by a receiver with collecting aperture $A_{r}$ and spectral bandwidth $\Delta \lambda$ is given by the following integral

$$
\begin{aligned}
& P_{a s}=\int_{h_{s}}^{\infty} d z N_{\lambda}^{0}(\Delta \lambda) \frac{A_{r}}{(R-z)^{2}} \frac{\beta(z)}{4 \pi}\left[\pi \theta_{r}^{2}(R-z)^{2}\right][T(z, \infty)]^{1+\sec \theta_{s}} \\
& =\frac{N_{\lambda}^{0}(\Delta \lambda) A_{r} \Omega_{r}}{4 \pi} \int_{h_{s}}^{\infty} d z \beta(z)[T(z, \infty)]^{1+\sec \theta_{s}}
\end{aligned}
$$

where $(R-z)$ is the range to the differential scattering volume, $\beta(z)$ is the volumetric scattering coefficient at a height $z$ above sea level, and we have assumed isotropic scattering. For wavelengths far from absorption lines, the atmospheric extinction is due entirely to scattering so we can set $\beta(z)=\mu(z)$. Using (Al) and (A4), the rate at which atmospheric solar scatter is converted into photoelectrons by the receiver is

$$
\begin{aligned}
& \dot{n}_{a s}=\frac{\eta_{q} \eta_{r}}{h v} \frac{N_{\lambda}^{0}(\Delta \lambda) A_{r} \Omega_{r}}{4 \pi} \int_{h_{s}}^{\infty} d z \beta(z)[T(z, \infty)]^{1+\sec \theta_{s}} \\
& =\frac{\eta_{q} \eta_{r}}{h v} \frac{N_{\lambda}^{0}(\Delta \lambda) A_{r} \Omega_{r}}{4 \pi} \int_{h_{s}}^{\infty} d z \mu(z) \exp \left[-\left(1+\sec \theta_{s}\right) \int_{z}^{\infty} d z^{\prime} \mu\left(z^{\prime}\right)\right]
\end{aligned}
$$

where $\eta_{q}$ is the detector quantum efficiency, $\eta_{\mathrm{x}}$ is the receiver optical throughput efficiency, and $h v$ is the photon energy at the laser wavelength. In order to evaluate the final integral in (A5), we define the new variable 


$$
\xi=\left(1+\sec \theta_{s}\right) \int_{z}^{\infty} d z^{\prime} \mu\left(z^{\prime}\right)
$$

which further implies

$$
d \xi=-\left(1+\sec \theta_{s}\right) \mu(z) d z
$$

Substituting (A6) into (A5) and evaluating the trivial integral yields

$$
\dot{n}_{a s}=\frac{\eta_{q} \eta_{r}}{h v} \frac{N_{\lambda}^{0}(\Delta \lambda) A_{r} \Omega_{r}}{4 \pi}\left\{\frac{1-T_{0}^{1+\sec \theta_{s}}}{1+\sec \theta_{s}}\right\}
$$

Note that as $T_{0} \rightarrow 1$ (no atmospheric scattering), the background count rate correctly goes to zero for all values of $\theta_{s}$.

When the solar zenith angle equals zero, the background photoelectron count rate due to atmospheric scatter has a maximum given by

$$
\dot{n}_{a s} \leq \frac{\eta_{q} \eta_{r} A_{r}}{4 \pi h v} N_{\lambda}^{0}(\Delta \lambda) \Omega_{r}\left\{\frac{1-T_{0}^{2}}{2}\right\}
$$

and goes to zero as $\theta_{\mathrm{s}} \rightarrow \pi / 2$.

\section{A.2 SOLAR SCATTER OFF THE SURFACE}

The mean background count rate due to solar scatter off a Lambertian surface is given by the equation

$$
\dot{n}_{s s}=\frac{\eta_{q} \eta_{r} A_{r}}{\pi h v} N_{\lambda}^{0}(\Delta \lambda) \Omega_{r} \rho T_{0}^{1+\sec \theta_{s}} \cos \psi
$$

where $\psi$ is the subtended angle between two vectors - the surface normal and a vector originating from the center of the beam pattem on the ground and directed at the Sun as in Figure 3. The angle $\psi$ can be expressed in terms of the local surface slope, $\sigma$, and the solar zenith angle, $\theta_{s}$, via the equation

$$
\cos \psi=\cos \sigma \cos \theta_{s}+\sin \sigma \sin \theta_{s} \cos \varphi
$$

where $\varphi$ is the angle between the plane containing the altimeter line-of-sight and the Sun $(x-z$ plane) and the plane containing the altimeter line-of-sight and the surface normal. Since $2 \pi \geq \varphi \geq 0$, we have the condition

$$
\cos \left(\sigma-\theta_{s}\right) \geq \cos \psi \geq \cos \left(\sigma+\theta_{s}\right)
$$


and $\cos \psi$ has the maximum value of 1 when $\varphi=0$ and $\sigma=\theta_{s}$. Thus, from (A9), the following inequality holds

$$
\dot{n}_{s s} \leq \frac{\eta_{q} \eta_{r} A_{r}}{\pi h v} N_{\lambda}^{0}(\Delta \lambda) \Omega_{r} \rho T_{0}^{2}
$$

Note from (A9) that the surface is in shadow (i.e. no scattered surface radiation) when $3 \pi / 2 \geq \psi$ $\geq \pi / 2$.

\section{APPENDIX B: EFFECTS OF RECEIVER DEAD TIMES ON SIGNAL CELL CONTRAST}

\section{B.1 RELEVANT TIME CONSTANTS}

There are several important time constants which can affect the overall performance of the microaltimeter receiver. These include:

$\tau_{s}$, the overall spreading of a single laser pulse by the surface under investigation

$\tau_{r}$, the integration time of the photodetector and front end receiver electronics which determines whether or not threshold is achieved in the timing discriminator

$\tau_{d}$, the "dead time" of the detector or range receiver in which no pulse timing can be performed following a triggering signal or noise count above threshold

$\tau_{\mathrm{g}}$, the range gate width

$\tau_{b}$, the duration of a range bin in the correlation range receiver

$\tau_{\mathrm{f}}$, the duration of a frame in the correlation range receiver

\section{B.2 GENERAL THEORY}

We define the generating function of the Poisson process in the usual way, i.e.

$$
g(t)=\lambda+s(t)
$$

where $\lambda$ is a noise background (assumed to be constant over the range gate width) and $s(t)$ is the signal waveform which is distributed over a time interval, $\tau_{s}$, which varies with surface slope and roughness. The mean number of counts occurring in an infinitesimal time interval $d t$ is given by

$$
d n(t)=g(t) d t
$$

which is also equal to the probability of a photon being detected in the infinitesimal interval $d t$ by a receiver with a detection threshold of one photoelectron. 
To obtain the true mean photon count within the differential time interval at time $t$, we must multiply (B.2) by the probability that no photon detection took place within the previous time interval $t-\tau_{d}<t^{\prime}<t$, i.e.

$P_{n d}\left(t-\tau_{d} \leq t^{\prime}<t\right)==\exp \left\{-\int_{t-\tau_{d}}^{t} d t^{\prime} g\left(t^{\prime}\right)\right\}$

Thus, the mean count in the mth range bin, is given by the integral

$n_{m}=\int_{(m-1) \tau_{b}}^{m \tau_{b}} g(t) \exp \left\{-\int_{t-\tau_{d}}^{t} d t^{\prime} g\left(t^{\prime}\right)\right\} d t$

If signal is not present in the range bin, we can set $s(t)=0$ in (B.4) and the mean number of noise counts collected in a range bin is reduced, relative to a detector/receiver with infinitely fast recovery, to

$n_{b}{ }^{\prime}=\lambda \tau_{b} \exp \left(-\lambda \tau_{d}\right)=n_{b} \exp \left(-\lambda \tau_{d}\right)$

Thus, for Mhz noise rates and receiver dead times on the order of $100 \mathrm{nsec}$ or less, the mean noise count per range bin is reduced by less than $10 \%$.

Substituting (B.1) into (B.4) yields the following general result for the mean count within the signal bin

$n_{\mathrm{t}}{ }^{\prime}=\exp \left(-\lambda \tau_{d}\right)\left\{\lambda \int_{(m-1) \tau_{b}}^{m \tau_{b}} d t \exp \left[-\int_{t-\tau_{d}}^{t} d t^{\prime} s\left(t^{\prime}\right)\right]+\int_{t_{1}}^{t_{2}} d t s(t) \exp \left[-\int_{t-\tau_{d}}^{t} d t^{\prime} s\left(t^{\prime}\right)\right]\right\}$

where we have assumed that the signal pulse is confined to the interval $t_{1}<t<t_{2}$ which is totally contained within the mth range bin.

The cell contrast in this very general case is now given by

$$
C_{d}=\frac{n_{t}{ }^{\prime}}{n_{b}{ }^{\prime}}=\frac{1}{n_{b}}\left\{\lambda \int_{(m-1) \tau_{b}}^{m \tau_{b}} d t \exp \left[-\int_{t-\tau_{d}}^{t} d t^{\prime} s\left(t^{\prime}\right)\right]+\int_{t_{1}}^{t_{2}} d t s(t) \exp \left[-\int_{t-\tau_{d}}^{t} d t^{\prime} s\left(t^{\prime}\right)\right]\right\}
$$

where the subscript $d$ stands for dead time. It can be easily seen that (B.5) through (B.7) approach the proper values in the limit as $\tau_{d} \rightarrow 0$, ie. $n_{b}^{\prime} \rightarrow n_{b}$ and $n_{t}^{\prime} \rightarrow n_{b}+n_{s}$ and that $C$ approaches the value given by Eq. (7a) in the main text. 


\section{B.3 SPECIAL CASE: $\tau_{b}>\tau_{d}>\tau_{s}$}

If the receiver dead time is longer than the surface return waveform but shorter than the range bin $\left(\tau_{b}>\tau_{d}>\tau_{s}=t_{2}-t_{1}\right)$, the second integral in (B.7) can be evaluated by a change of variable, i.e.

$y=\int_{t-t_{d}}^{t} d t^{\prime} s\left(t^{\prime}\right)=\int_{t_{1}}^{t} d t^{\prime} s\left(t^{\prime}\right)$

so that

$$
\int_{t_{1}}^{t_{2}} d t s(t) \exp \left[-\int_{t_{1}}^{t} d t^{\prime} s\left(t^{\prime}\right)\right]=\int_{0}^{n_{s}} d y \mathrm{e}^{-y}=1-e^{-n_{s}}
$$

To evaluate the first integral in (B.7), we first break the range bin up into four time segments (1before the signal pulse; 2 -during the pulse; 3 -immediately after the pulse while the detector or receiver is recovering; and 4 - following detector or receiver recovery). We obtain

$$
\begin{aligned}
& \lambda \int_{(m-1) \tau_{b}}^{m \tau_{b}} d t \exp \left[-\int_{t-\tau_{d}}^{t} d t^{\prime} s\left(t^{\prime}\right)\right] \\
& =\lambda\left\{\left(\tau_{b}-\tau_{s}-\tau_{d}\right)+\int_{t_{1}}^{t_{2}} d t \exp \left[-\int_{t_{1}}^{t} d t^{\prime} s\left(t^{\prime}\right)\right]+\int_{t_{2}}^{t_{2}+\tau_{d}} d t \exp \left[-\int_{t-\tau_{d}}^{t_{2}} d t^{\prime} s\left(t^{\prime}\right)\right]\right\}
\end{aligned}
$$

Using integration by parts [Selby, 1969], we can write for the first integral in (B.9)

$$
\begin{aligned}
& \int_{t_{1}}^{t_{2}} d t \exp \left[-\int_{t_{1}}^{t} d t^{\prime} s\left(t^{\prime}\right)\right]=\left(t_{2} e^{-n_{s}}-t_{1}\right)+\int_{t_{1}}^{t_{2}} d t t s(t) \exp \left[-\int_{t_{1}}^{t} d t^{\prime} s\left(t^{\prime}\right)\right] \\
& \cong\left(t_{2} e^{-n_{s}}-t_{1}\right)+\frac{t_{1}+t_{2}}{2}\left(1-e^{-n_{s}}\right)=\frac{\tau_{s}}{2}\left(1+e^{-n_{s}}\right)
\end{aligned}
$$

where we have used the fact that the signal retum is a sharp spike within the range bin to pull the factor of $t$ out of the integral in (B.10), leaving us with the integral in (B.8b).

We can perform a similar analysis for the second integral in (B.9) to obtain

$$
\begin{aligned}
& \int_{t_{2}}^{t_{2}+\tau_{d}} d t \exp \left[-\int_{t-\tau_{d}}^{t_{2}} d t^{\prime} s\left(t^{\prime}\right)\right]=\left[\tau_{d}+t_{2}\left(1-e^{-n_{s}}\right)\right]-\int_{t_{2}}^{t_{2}+\tau_{d}} d t t s\left(t-\tau_{d}\right) \exp \left[-\int_{t-\tau_{d}}^{t_{2}} d t^{\prime} s\left(t^{\prime}\right)\right] \\
& \cong \frac{\tau_{s}}{2}\left(1-e^{-n_{s}}\right)+\tau_{d} e^{-n_{s}}
\end{aligned}
$$


Substituting (B.10) and (B.11) into (B.9) and inserting the result along with (B8.b) into (B.7) gives the following simple expression for the signal cell contrast in the presence of a detector or receiver dead time

$$
C_{d}=1+\left(1-e^{-n_{s}}\right)\left[\frac{1}{n_{b}}-\frac{\tau_{d}}{\tau_{b}}\right]
$$

\section{REFERENCES}

Abbott, R., Shelus, P., 1973, Laser observations of the Moon: Identification and construction of normal points for 1969-1971, The Astronomical Journal 78, pp. 784-793.

Abshire, J., Ketchum, E., Afzal, R., Millar, P., Sun, X., 2000, The Geoscience Laser Altimeter System (GLAS) for the ICEsat Mission, Proc. CLEO/QELS 2000, San Francisco, CA, May 7-12 (to be published).

Aharonson, O., 2000, Massachusetts Institute of Technology, private communication.

Charboneau, S., Allard, L., Young, J., Dyck, G., Kyle, B., 1992, Two-dimensional time resolved imaging with 100 ps resolution using a resistive anode photomultiplier tube, Review of Science Instruments 63, pp. 5315-5319.

Cova, S., Ghioni, M., Lacaita, A., Samori, C. Zappa, F., 1996, Avalanche photodiodes and quenching circuits for single photon detection, Applied Optics, vol. 35, pp 1956-1976.

Degnan, J., McGarry, J., 1997, SLR2000: Eyesafe and autonomous single photoelectron satellite laser ranging at kilohertz rates, SPIE Proceedings 3218, pp. 63-77.

Degnan, J., 1998, SLR2000 Project: Engineering overview and status, Proc. $11^{\text {th }}$ Int. Workshop on Laser Ranging, vol. 2, pp. 389-398, Deggendorf, Germany, Sept. 20-25.

Degnan J., McGarry, J., 1998, Feasibility study of multikilohertz spaceborne microlaser altimeters, European Geophysical Society (EGS) Annual Symposium, Nice, France, April 20-24. (Abstract: Annales Geophysicae, Part I Society Symposia, Solid Earth Geophysics and Geodesy, Supplement 1 to Volume 16, p. C379)

Degnan, J., Steggerda, C., Karger, A., Zagwodzki, T., 1999, High quantum efficiency visible detector array integrated with a compact subnanosecond range receiver, Proposal submitted to NASA Research Announcement NRA 99-OSS-05, Advanced Cross Enterprise Technology Development for NASA Missions, February.

Dubayah, R., Blair, J.B., Bufton, J.L., Clark, D. B. JaJa, J., Knox, R., Luthcke, S. B., Prince, S., Weishampel, J., 1997, The Vegetation Canopy Lidar Mission, Proceedings: Land Satellite Information in the Next Decade II: Sources and Applications, 1997, American Society for Photogrammetry and Remote Sensing, Bethesda, MD, December 1997.

Ebstein, S., Cone, P., Ku, J., Prototype Solar-Blind UV Sensitive PAPA Photon-Counting Detector, Final Report for Phase II SBIR NAS5-33222, Lexitek Inc., 14 Mica Lane \#6, Wellesley MA 02481 USA.

Ho, C., Priedhorsky, W., 1995, Volumetric measurement of plant canopies using laser ranging and mapping technique with a photon counting detector, Los Alamos National Laboratory, Internal Memorandum, NIS-2-95-206, Sept. 12, 1995.

Priedhorsky, W., Smith, R., Ho, C., 1996, Laser ranging and mapping with a photon counting detector, Applied Optics 35, pp.441-452.

Ramos-Izquierdo, L., Bufton, J., Hayes, P., 1994, "Optical system design and integration of the Mars Observer Laser Altimeter", Applied Optics, vol. 33, pp. 307-322.

Stitch, M., 1972, Laser Rangefinding, in Laser Handbook 2, Eds. F. T. Arecchi and E. O. SchulzDubois, North Holland Publishing Company, Amsterdam, pp. 1745- 1804. 
Selby, S., 1969, CRC Standard Mathematical Tables (1 $7^{\text {th }}$ Student Edition), The Chemical Rubber Company, Cleveland OH, pp.400-402.

Titterton, P., Sweeney, H., Leonard, D., 1998, "System/Usage Impact of Operating the SLR2000 at $2 \mathrm{kHz}$ ", Proc. 11 th International Workshop on Laser Ranging, Deggendorf, Germany, pp. 426-437, September 21-25.

Vasile, S., Gothoskar, P., Farrell, R., Sdrulla, D., 1997, "Photon detection with high gain avalanche photodiode arrays", IEEE Trans. Nuclear Science, vol. 45, pp. 720-723.

Zayhowski, J., Passively Q-switched microchip lasers and applications, Rev. Laser Eng., vol. 26, pp. 841-846, 1998. 


\section{FIGURE CAPTIONS:}

Figure 1: Normalized surface retum rate $\left(f_{R} / f_{\text {max }}\right)$ as a function of mean signal strength, $n_{s}$, and three detector thresholds, $\mathrm{n}_{\mathrm{t}}=1,2$, and 3 photoelectrons (pe). Conventional laser altimeters typically operate with high signal strengths and high noise thresholds (lower right of the asymptotic curve) and therefore make inefficient use of the available laser photons whereas photon-counting microaltimeters operate on the most efficient $n_{\mathfrak{t}}=1$ curve at low mean signal counts $\left(n_{s} \leq 1\right)$.

Figure 2: Principles behind a correlation range receiver. The range gate is divided into equal duration range bins, and several consecutive laser fires are combined to form a frame. The 2-D area bounded by the range bin and frame dividing lines is a cell. Multiple frames form a superframe. Photon counts are accumulated within each cell and, if the total count $K$ exceeds the frame threshold $\mathrm{K}_{\text {opt }}$, the cell is identified as signal; otherwise it is tentatively identified as noise. A valid trajectory is one in which the signal cell is not displaced by more than one range bin in consecutive frames. Applying this criteria in algorithms which look forward and backward in time and $N$ of $M$ techniques can help recover lost or missing data in near real time.

Figure 3: Definitions of angles used in the solar background noise analysis.

Figure 4: Plots of (a) the Differential Cell Count normalized to $M$, (b) the probability of correctly identifying the signal cell in a frame, and (c) the mean number of cells incorrectly identified as signal within a frame as a function of the mean signal counts in a frame, $N_{s}$, for $N_{b i n}=500$ and four contrast values, $C=2$ (solid), 3 (dot), 5 (dash), and 10 (dot-dash). Note that different combinations of $C$ and $N_{s}$ give essentially equivalent performance, but systems with low contrast require more signal counts per frame (longer frame times) to achieve the same level of discrimination.

Figure 5: Block diagram of the developmental NASA Airborne Multi-kilohertz Microlaser Altimeter Instrument.

Figure 6: Analysis summary of NASA Airbome Multi-kilohertz Microlaser Altimeter Instrument operating at a $10 \mathrm{kHz}$ rate from a cruise altitude of $40,000 \mathrm{ft}(12 \mathrm{~km})$ : (a) Maximum surface sampling rate vs power-aperture product; (b) signal cell contrast vs frame interval; (c) optimum frame threshold vs frame interval; (d) normalized differential cell count vs frame interval; (e) probability of successfully detecting signal cell vs frame interval; and (f) mean number of falsely acquired noise cells per frame vs frame interval. The three curves in each figure correspond to different surface reflectances, i.e. $\rho=1$ (solid), 0.15 (dot), 0.10 (dash).

Figure 7: Microaltimeter performance over simulated barren terrain containing a $50 \mathrm{~m}$ cliff: (a) coarse scale showing the full 4 microsecond range gate; (b) fine scale showing points tentatively identified as terrain by the signal extraction algorithm over a short segment on the cliff.

Figure 8: The following Mars microaltimeter parameters are plotted versus the mean signal count per laser fire, $n_{\mathbf{s}}$ : (a) single shot probability of detection for 1 pe threshold; (b) required laser fire rate to achieve $3 \mathrm{kHz}$ sampling rate from worst case surface; (c) receive telescope diameter; (d) transmitted laser energy; (e) average transmitter power; and (f) the Power-Aperture Product. The four curves in (c) through (e) correspond to different assumed contrast values, i.e. $C=2$ (solid), 3 (dot), 5(dash), and 10 (dot-dash). For each contrast, the corresponding value of the mean signal count per frame, $N_{s}$, is given by Table 5 to ensure equivalent performance. In $(f)$, all four curves overlap. 
Figure 9: Block diagram of an all-digital modulo timer integrated with a 6x6 micro-APD array. 


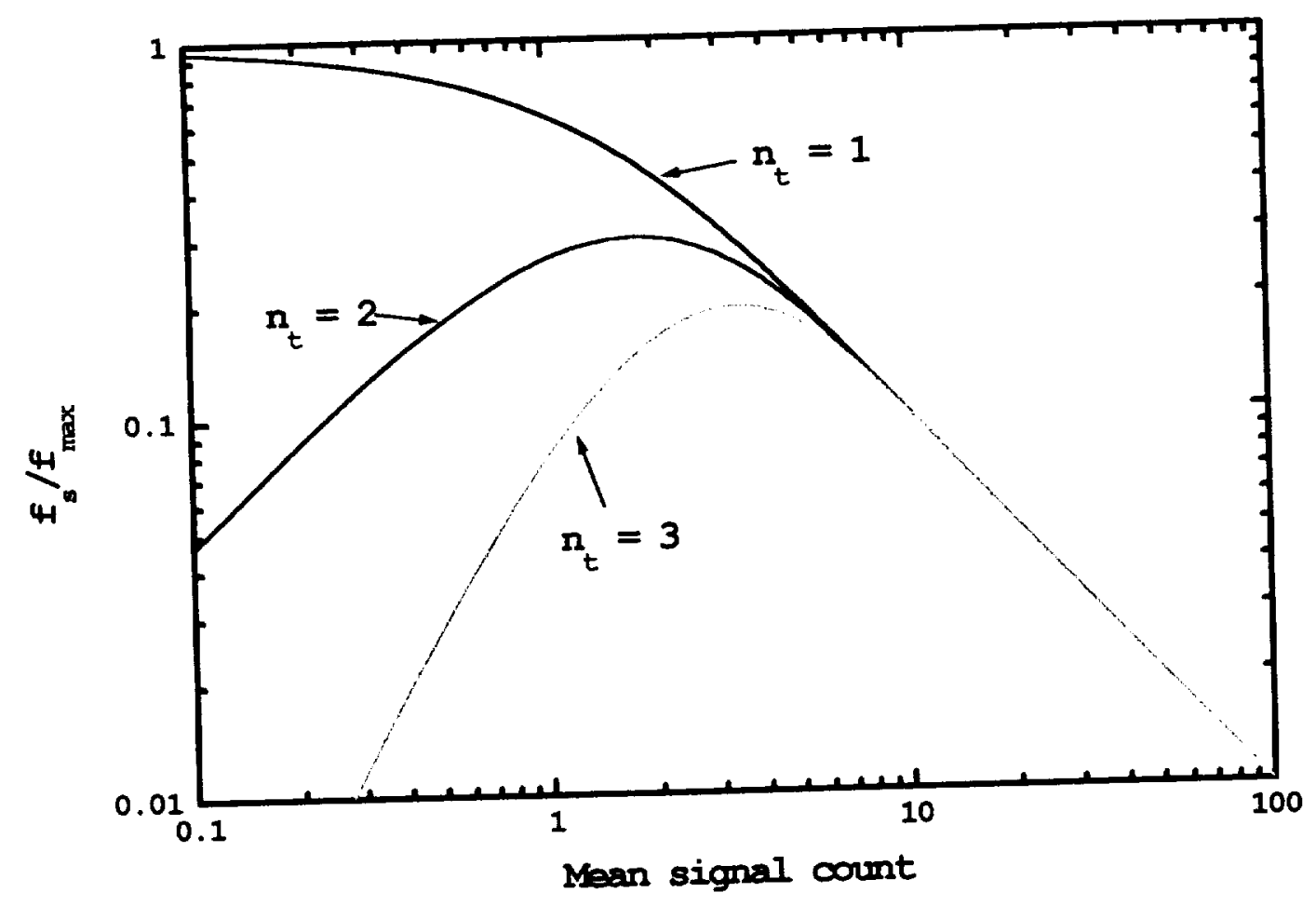

Figure 1 


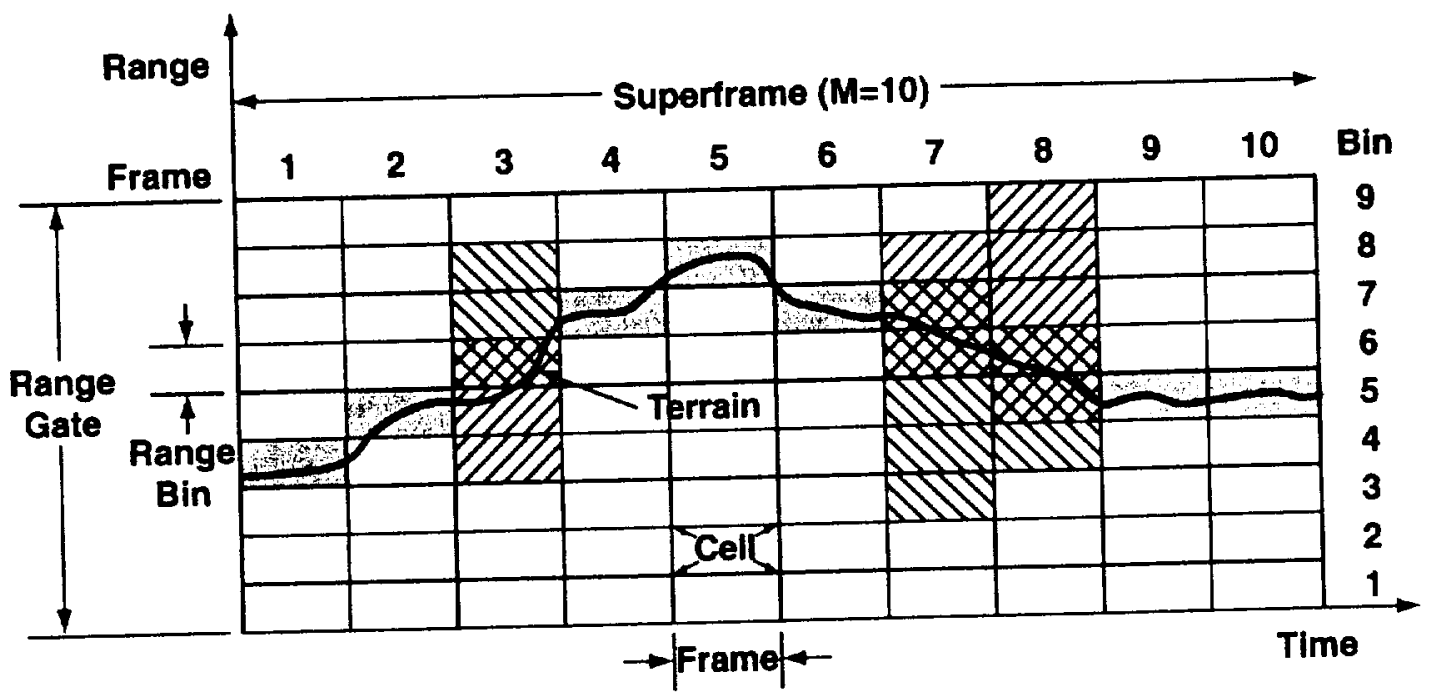

\begin{tabular}{|c|c|c|c|c|}
\hline 17\% & & $E D$ & & \\
\hline $\begin{array}{l}\text { Slgnal } \\
\text { Cell } \\
K>K_{\text {opt }}\end{array}$ & $\begin{array}{c}\text { Nolse } \\
\text { Cell } \\
K<K_{\text {opt }}\end{array}$ & $\begin{array}{c}\text { Forward } \\
\text { Candidate } \\
\text { Cell } \\
\mathrm{K}_{<} \mathrm{K}_{\text {opt }}\end{array}$ & $\begin{array}{c}\text { Backward } \\
\text { Candidate } \\
\text { Cell } \\
K<K_{\text {opt }}\end{array}$ & $\begin{array}{c}\text { Forward } \\
\text { Backward } \\
\text { Cell } \\
K<K_{\text {opt }}\end{array}$ \\
\hline
\end{tabular}

Figure 2 


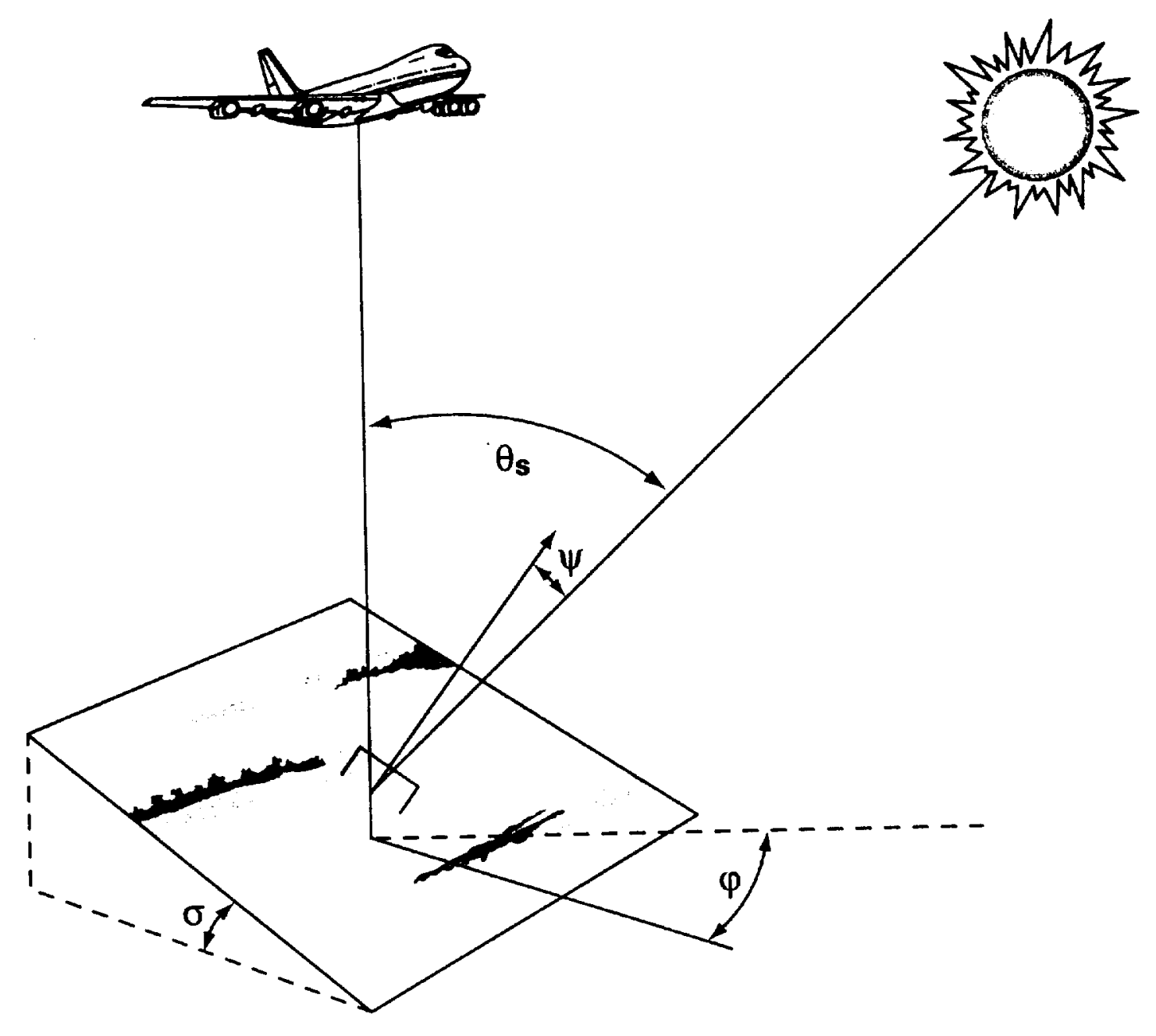

Figure 3 


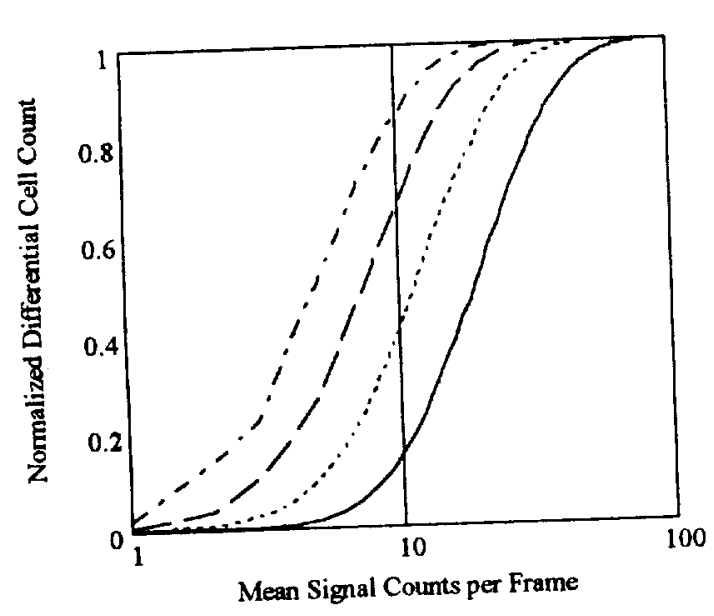

(a)

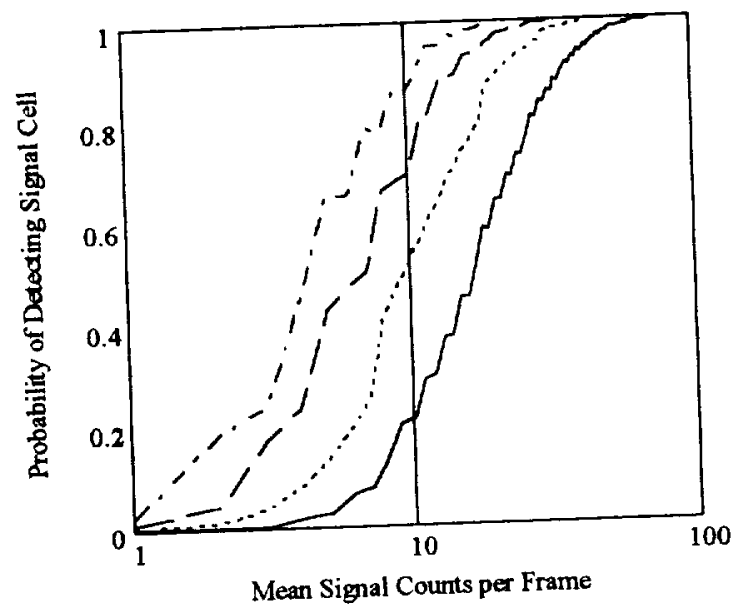

(b)

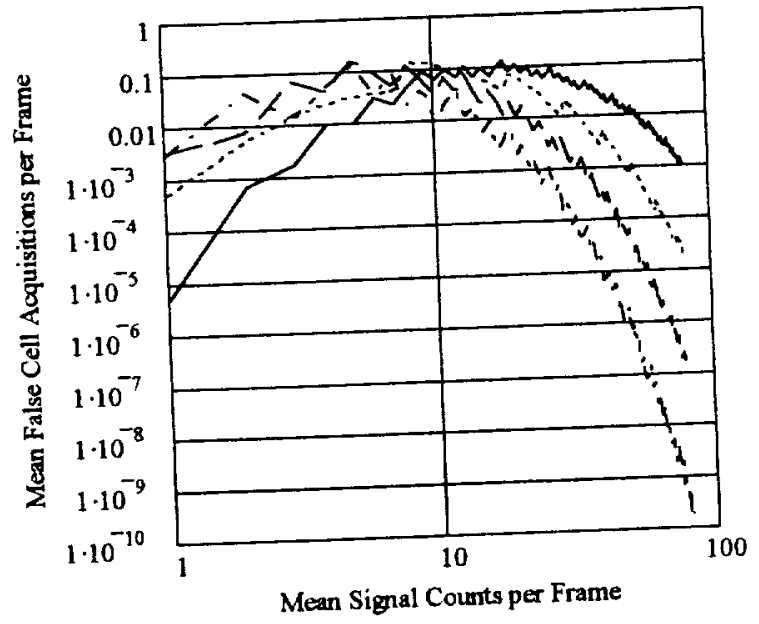

(c)

Figure 4 


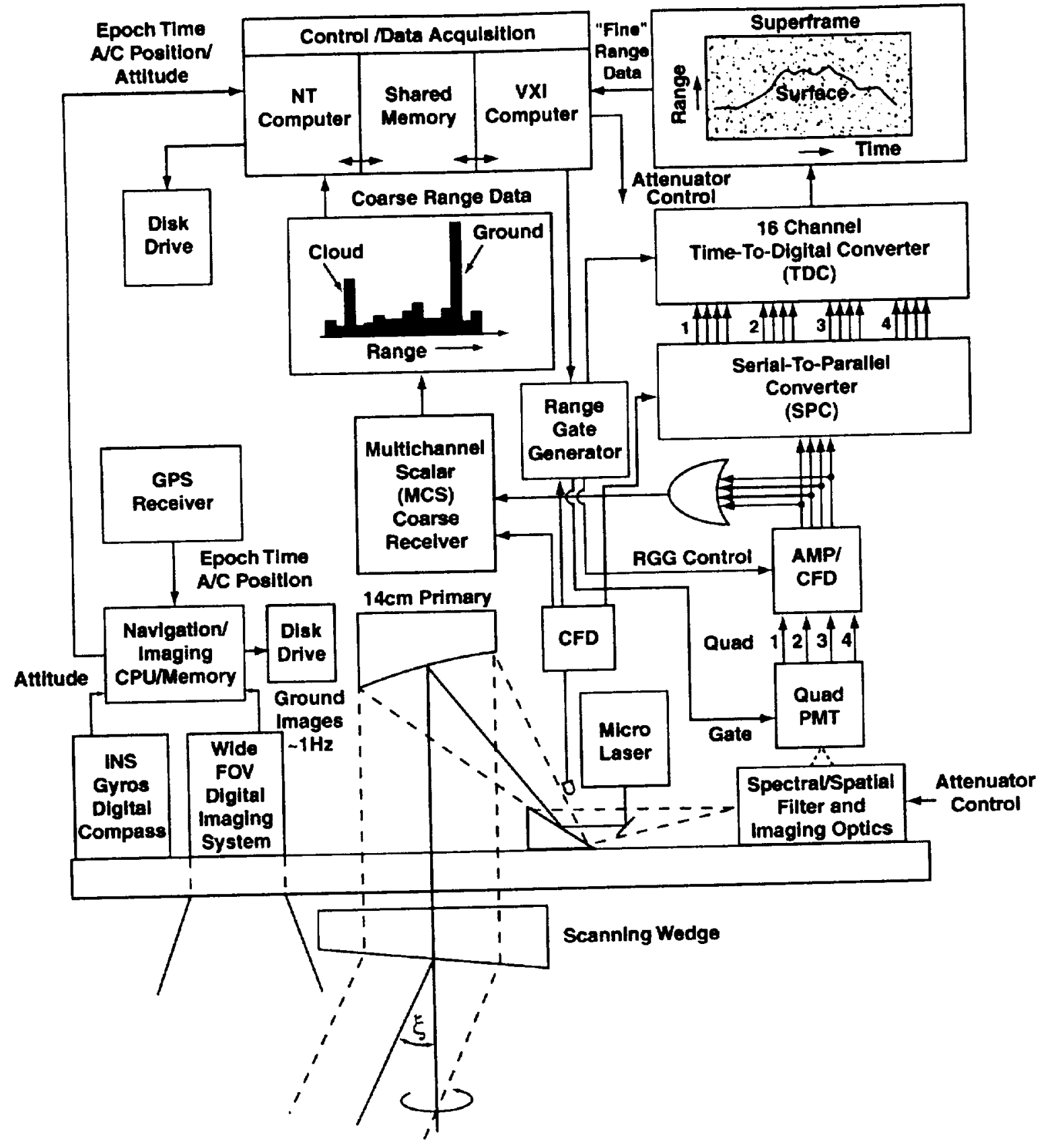

Figure 5 


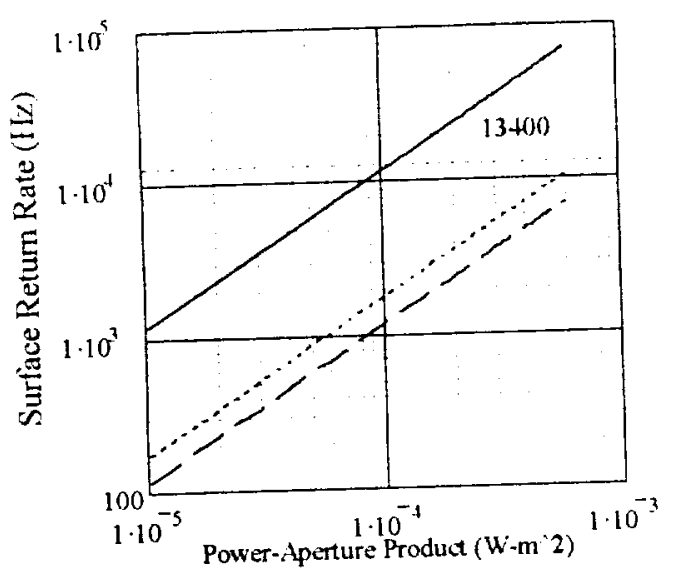

(a) Surface Returns vs Power-Aperture

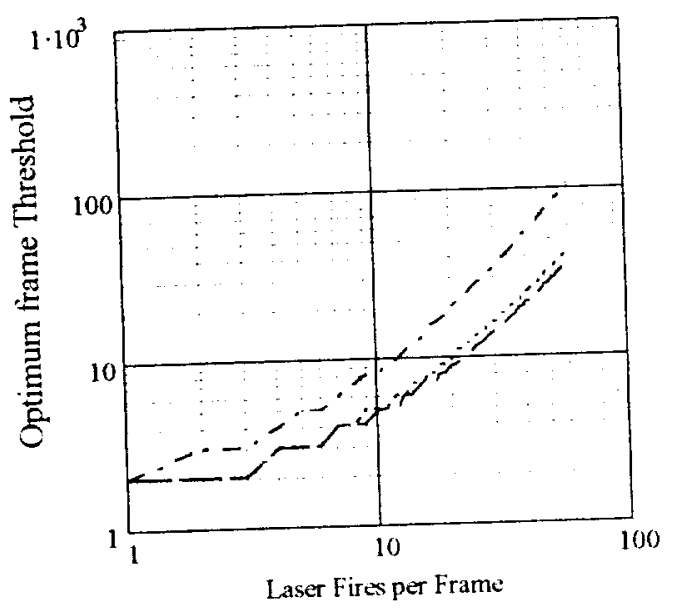

(c) Optimum Frame Threshold

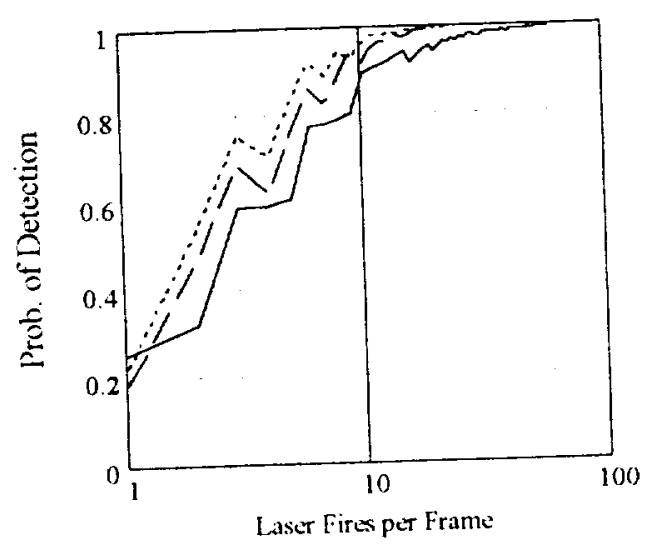

(c) Prob. of Detecting Signal Cell

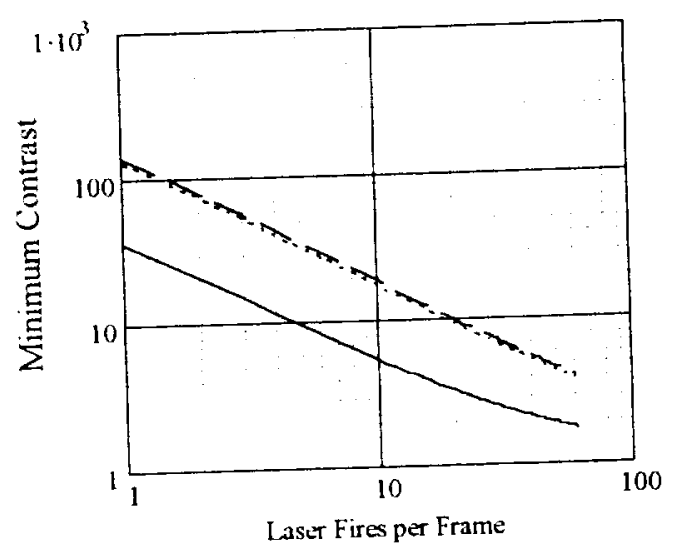

(b) Minimum Contrast vs Frame Time

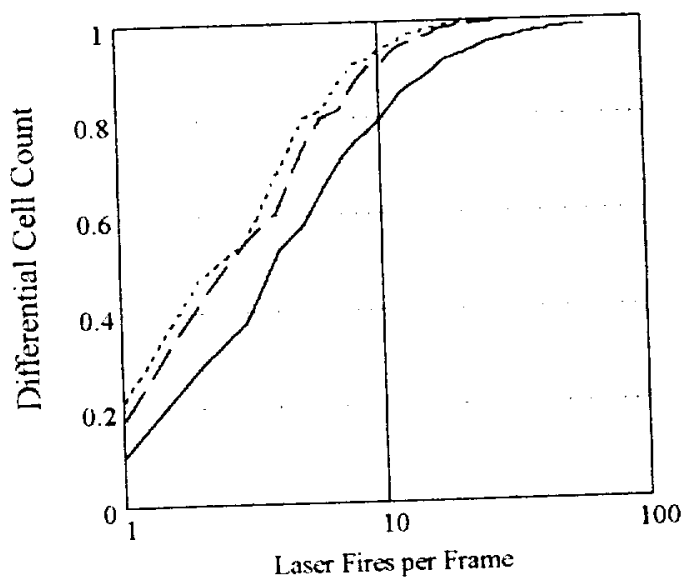

(d) Normalized Differential Cell Count

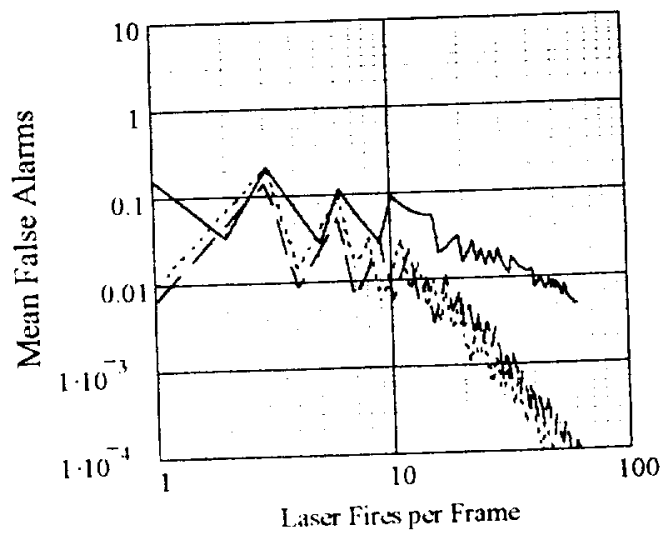

(f) Mean False Alarms per Frame

Figure 6 


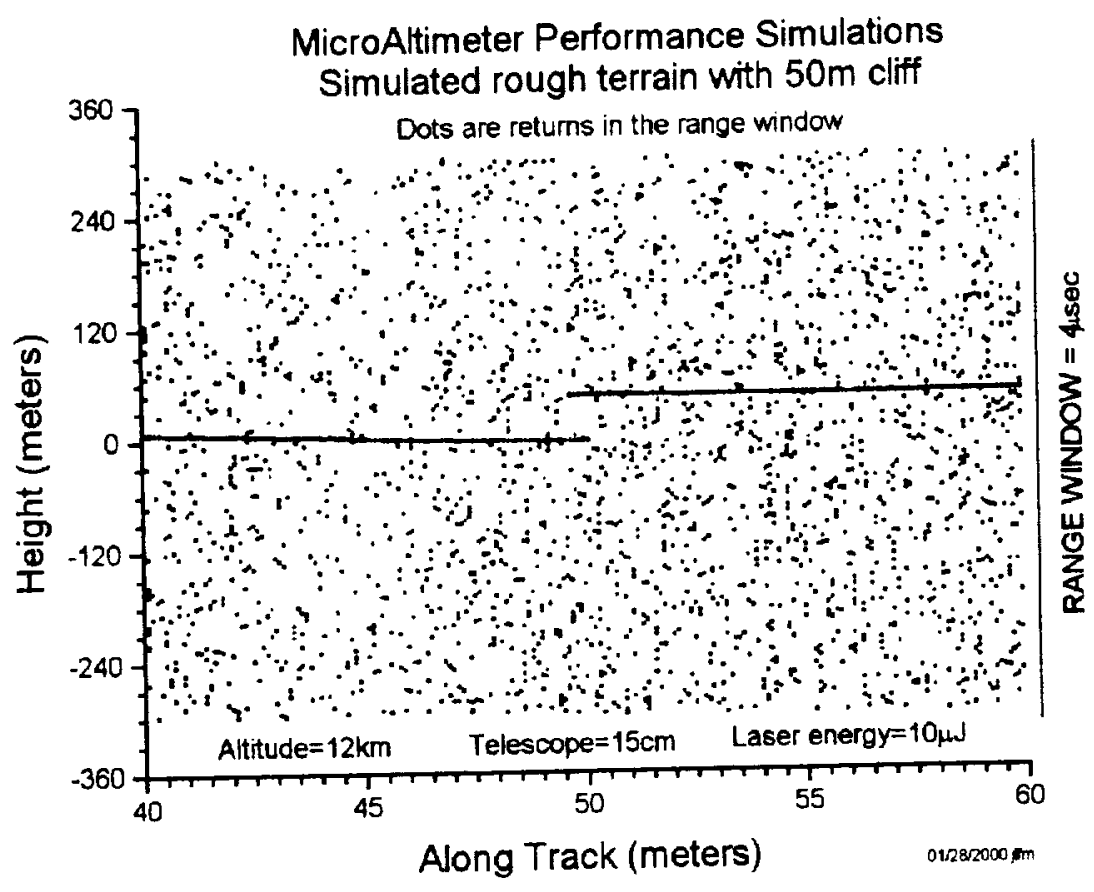

(a)

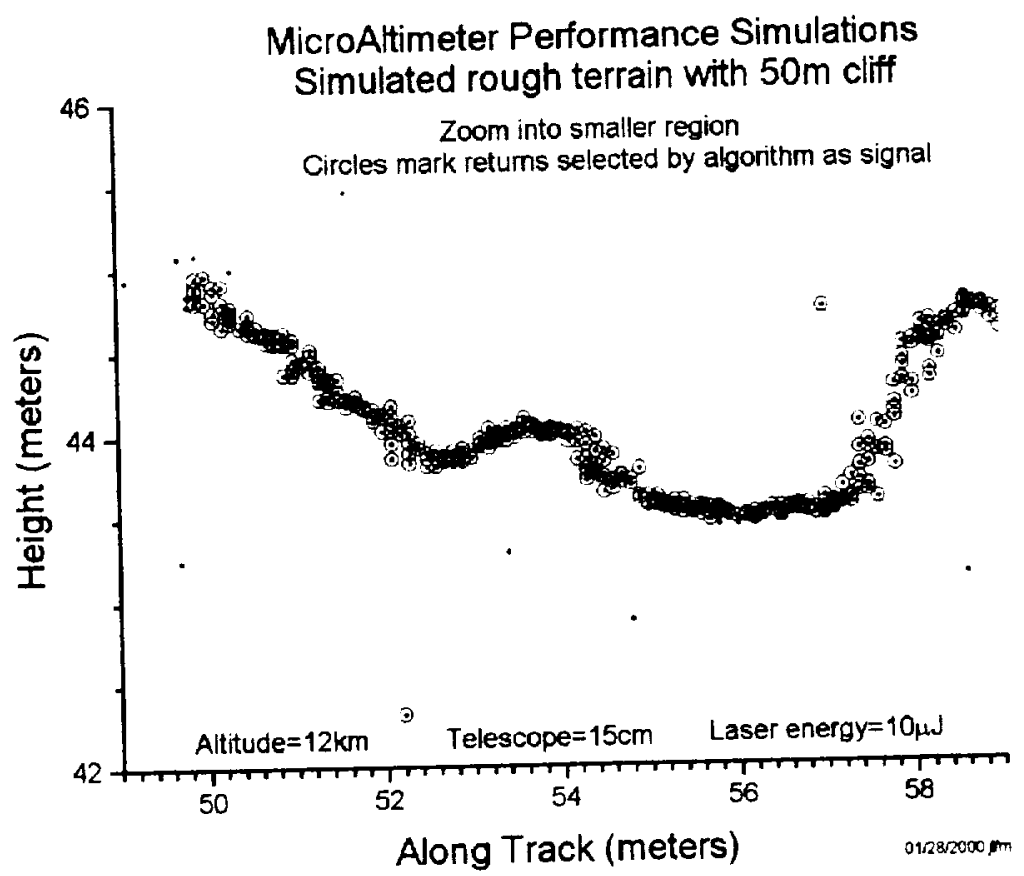

(b)

Figure 7 


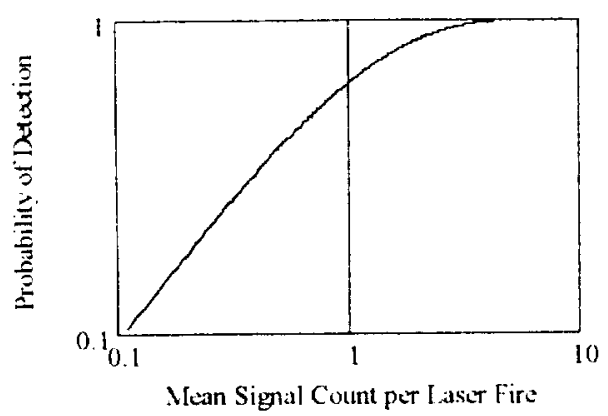

(a) Single Shot Probability of Detection

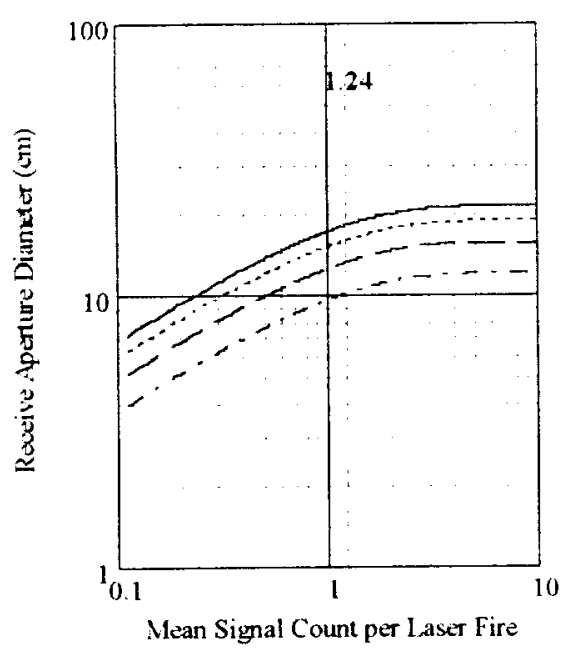

(c) Receive Aperture Diameter $(\mathrm{cm})$

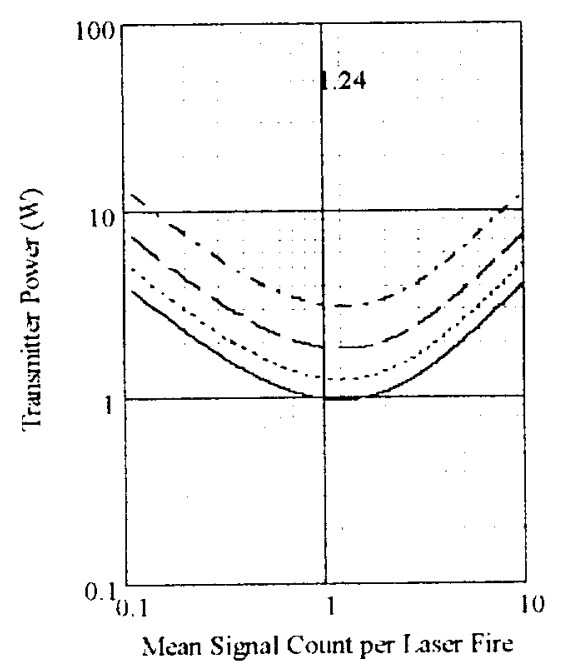

(c) Transmitter Power (Watts)

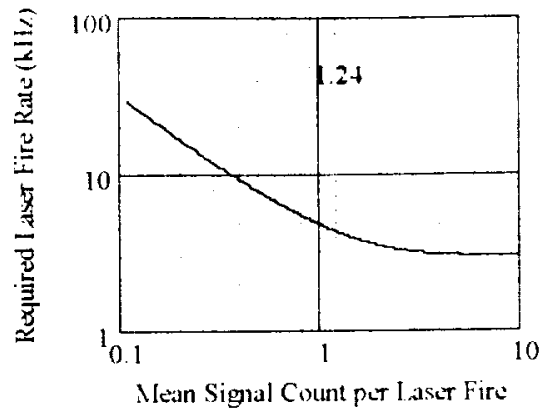

(b) Required Laser Fire Rate

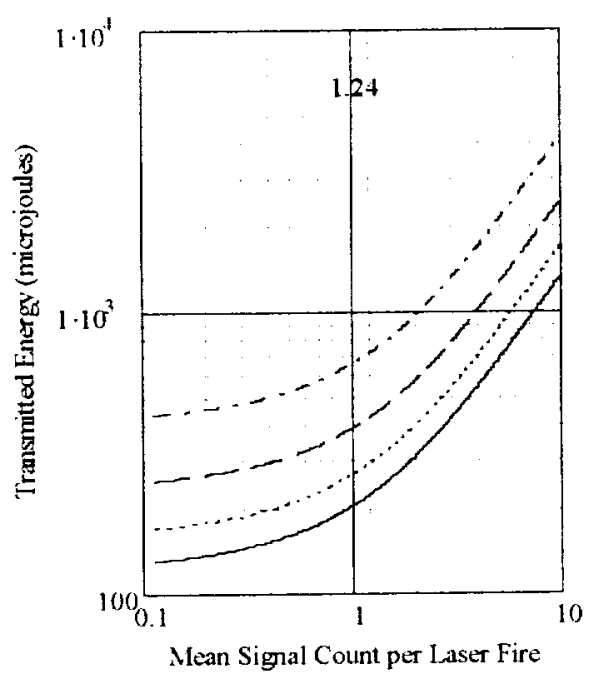

(d) Transmitted Energy (microjoules)

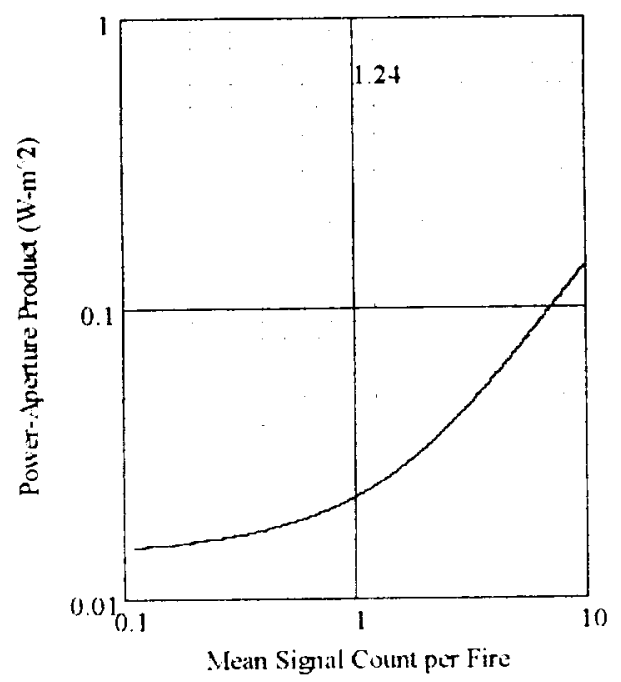

(f) Power- $\wedge$ perture Product $\left(W-\mathrm{m}^{\wedge} 2\right)$

Figure 8 


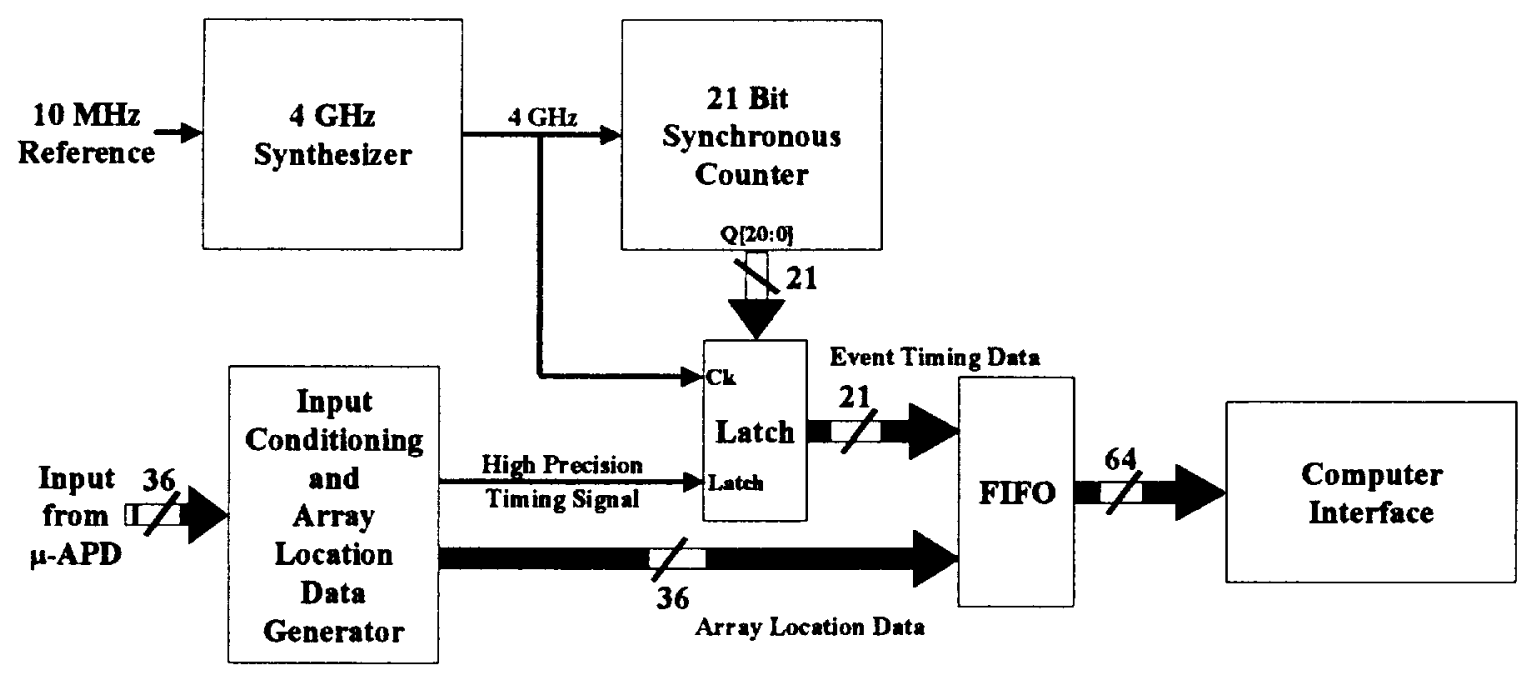

Figure 9 